\title{
Mature parvalbumin interneuron function in prefrontal cortex requires activity during a postnatal sensitive period
}

Sarah E. Canetta ${ }^{1,3,6^{*}}$, Emma S. Holt ${ }^{3}$, Laura J. Benoit ${ }^{1,3}$, Eric Teboul ${ }^{3}$, R. Todd Ogden ${ }^{5}$, Alexander Z. Harris ${ }^{1,4,7}$ \& Christoph Kellendonk ${ }^{1,2,3,7^{*}}$

${ }^{1}$ Department of Psychiatry, ${ }^{2}$ Department of Pharmacology, Columbia University Medical Center, New York, NY 10032, USA

${ }^{3}$ Division of Molecular Therapeutics, ${ }^{4}$ Division of Integrative Neuroscience, New York State Psychiatric Institute, New York, NY 10032, USA

${ }^{5}$ Department of Biostatistics, Mailman School of Public Health, Columbia University Medical Center, New York, NY 10032, USA

${ }^{6}$ Lead contact; Further information and requests for resources and reagents should be directed to and will be fulfilled by the lead contact, Sarah E. Canetta (ses2119@cumc.columbia.edu)

${ }^{7}$ Senior author

*Correspondence: ses2119@cumc.columbia.edu \& ck491@cumc.columbia.edu 


\section{Summary}

Sensitive periods in which experience-driven changes in activity persistently shape circuit function are welldescribed in sensory cortex. Whether comparable periods govern the development of associative cortical areas, like the prefrontal cortex, remains unclear. Here, we focus on the role of activity in the maturation and circuit integration of prefrontal parvalbumin-expressing interneurons, as these cells play an essential role in sensory cortical maturation and develop in lockstep with overall prefrontal circuit function. We found that transiently decreasing prefrontal parvalbumin activity during peripubertal and adolescent development results in persistent impairments in adult functional connectivity, in vivo network function and set-shifting behavior that can be rescued by targeted activation of these interneurons in the adult animal. In contrast, comparable adult inhibition had no lasting effects. These findings identify an activity-dependent sensitive period for prefrontal parvalbumin maturation and highlight how abnormal parvalbumin activity early in life can persistently alter adult circuit function and behavior.

\section{Keywords}

Prefrontal cortex, development, parvalbumin, interneurons, gamma oscillations, cognitive flexibility, set-shifting, electrophysiology 


\section{Introduction}

The prefrontal cortex (PFC) is essential to many fundamental cognitive behaviors. Moreover, alterations in prefrontal function have been observed in multiple neurodevelopmental disorders characterized by impairments in cognitive function including schizophrenia and attentional deficit hyperactivity disorder (Brown and Tait, 2016). Evidence from lesion, functional imaging and transcranial magnetic stimulation studies in humans indicate that the PFC supports cognitive flexibility, such as the ability to switch between attentional sets (Boschin et al., 2017, Smith and Jonides, 1999, Glascher et al., 2019). Attentional set-shifting can be measured using tasks that require the subject to shift attention between different dimensions of a stimulus during ongoing testing in accordance with changing reinforcement contingencies. For example, in the Wisconsin Card Sorting Test, the initial discriminatory stimulus dimension that predicts outcome might be shapes on the card, followed by a shift to a different dimension such as color or number (and shape becomes irrelevant). Similar tasks measure set-shifting in rodents, although they are often odor and texture-based, rather than visual (Birrell and Brown, 2000, Bissonette et al., 2008, Cho et al., 2015). A shift in attention between stimulus dimensions is known as extradimensional (ED) set-shifting, and has been shown in both humans and rodents to rely on the PFC (Brown and Tait, 2016).

Experimental work in rodents has shown that within the PFC, GABAergic interneurons that express the marker parvalbumin support ED set-shifting (Canetta et al., 2016, Cho et al., 2015, Goodwill et al., 2018, Cho et al., 2020, Mukherjee et al., 2019). Prefrontal parvalbumin-expressing (PFC PV) interneurons are characterized by their fast and synchronous firing, as well as their innervation of the perisomatic region of pyramidal projection neurons (Markram et al., 2004, Wonders and Anderson, 2006). Both features allow tight control over cortical output (Markram et al., 2004). PFC PV interneuron activity contributes to fast fluctuations in neural activity termed gamma oscillations that are enhanced under conditions of increased cognitive load (Uhlhaas and Singer, 2010, Cardin et al., 2009, Sohal et al., 2009, Hu et al., 2014). This modulation points to a role for these interneurons in cognitive functioning. Indeed, rodent studies have directly linked decreases in PFC PV interneuron activity to impairments in ED attentional set-shifting (Canetta et al., 2016, Cho et al., 2015, Goodwill et al., 2018, Mukherjee et al., 2019, Cho et al., 2020).

Prefrontal PV interneurons undergo a protracted period of physiological maturation and integration into cortical circuitry during peripuberty and adolescence, prior to attaining their mature phenotype (Yang et al., 2014, Miyamae et al., 2017). Such dynamic, developmental windows often prove to be sensitive periods, defined as windows in which alterations in experience can result in long-lasting effects on the development of brain circuitry, physiology and behavior. The study of sensitive periods in cortical development has predominantly focused on sensory regions, such as the visual or somatosensory cortex. Environmental input, conveyed in the form of changes in activity, regulates the refinement of excitatory and inhibitory brain circuitry during sensitive periods in sensory cortices (Zhang and Poo, 2001, Antonini and Stryker, 1993, Baho and Di Cristo, 2012, Chattopadhyaya et al., 2007, Fu et al., 2012, Wu et al., 2012). In particular, the activity of PV interneurons during 
a developmental sensitive period can impact their integration into cortical circuitry (Baho and Di Cristo, 2012, Chattopadhyaya et al., 2007, Fu et al., 2012, Wu et al., 2012). However, it remains an open question whether there are sensitive periods in which changes in activity govern the maturation and functional integration of PV interneurons into prefrontal circuitry, in turn enabling the emergence of mature cognitive capabilities such as ED set-shifting.

Comparable studies of sensitive periods in the PFC have been hampered due to the difficulty of reversibly modulating incoming activity experienced by PV cells in vivo as the region receives no direct sensory input. To address this challenge, we have developed an approach that allows transient and reversible modulation of PFC PV interneuron activity during distinct developmental windows using viral expression of the DREADD receptor, hM4DG ${ }_{i}$, selectively in PFC PV interneurons (Roth, 2016). Using this approach, we have found that transiently decreasing PFC PV activity during peripubertal and adolescent development (postnatal day P14-P50), produces persistent impairments in adult ED set-shifting behavior, with corresponding deficits in task-evoked gamma oscillations and PV mediated inhibition. This juvenile period was a sensitive period for prefrontal circuit maturation as adult manipulations of PFC PV cells did not induce long-lasting changes in behavior and electrophysiology. Strikingly, we could rescue the cognitive behavior of developmentally silenced mice by acutely enhancing PFC PV excitability in adulthood. This demonstrates that cognitive impairments can be rescued even in the context of a developmentally altered brain.

Together, this work provides evidence of an activity-dependent sensitive period in prefrontal network maturation. These findings highlight how abnormal early experiences can increase risk for later maladaptive circuit function and behavior and provide hope that targeted manipulations can reverse developmentally-induced cognitive deficits. 


\section{Results}

\section{A chemogenetic system to transiently inhibit PFC PV interneurons during development or adulthood}

In order to transiently inhibit PFC PV interneuron activity during discrete windows in development or adulthood we expressed the DREADD receptor, $h M 4 D G_{i}$, specifically in prefrontal PV-expressing interneurons from a young age using a viral and genetic approach. Postnatal day 10 (P10) pups expressing Cre recombinase under the PV promoter were injected with an adeno-associated virus (AAV) carrying either a Cre-dependent form of

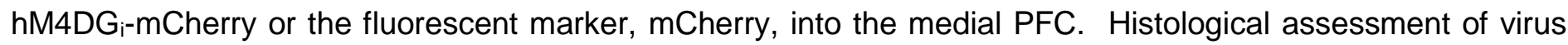
expression verified that hM4DGi-mCherry was restricted to PV-expressing cells predominantly in the PFC (Figure $1 A-B)$. To verify the functional effects of $h M 4 D G_{i}$ on $P V$ interneurons, slice electrophysiological recordings were made in hM4DGi-mCherry injected mice at P35 and P115 (Figure 1C, time points chosen to be in the middle of the developmental and adult manipulation windows, respectively). Prefrontal PV cells were identified under fluorescence based on their expression of mCherry. Resting membrane potential and firing frequency as a function of input current was recorded in these cells at baseline and following bath application of $10 \mu \mathrm{M}$ clozapine$\mathrm{n}$-oxide (CNO). Bath application resulted in a hyperpolarization of the resting membrane potential from baseline in both P35 and P115 mice expressing hM4DG, but not in PV cells from mice only expressing mCherry (Figure 1D-E; n=8 P35 \& P115 PV-mCherry cells, 7 P35 PV-hM4DGi cells, 6 P115 PV-hM4DG i cells, 1-way ANOVA, effect of treatment $F(2,18)=4.344, p=0.0289$; Holm-Sidak post-hoc, P35 PV-hM4DGi v mCherry * $p=0.035$, P115 $P V-h M 4 D G_{i} \vee m C h e r r y{ }^{*} p=0.0297$ ). Firing frequency as a function of input current was right-shifted following bath application of CNO to cells expressing hM4DG; at both P35 (Figure 1F; n=6 P35 PV-mCherry cells, 6 P35 PV-hM4DGi cells, 2-way ANOVA, effect of input current $F(29,540)=87.15 p<0.0001$ and effect of treatment $F(3,540)=15.43$ p<0.0001; Holm-Sidak post-hoc, P35 PV-hM4DG; CNO v P35 PV-hM4DG; $A C S F{ }^{* *} p=0.0013$, P35 PV-hM4DG CNO v PV-mCherry ACSF ${ }^{* * * *} \mathrm{p}<0.0001$, P35 PV-hM4DG CNO v PV-mCherry CNO ${ }^{* * *} \mathrm{p}<0.0001$ ) and P115 (Figure 1G; n=5 P115 PV-hM4DGi cells, 2-way ANOVA, effect of input current $F(19,160)=49.54 p<0.0001$ and effect of treatment $\left.F(1,160)=10.52{ }^{* *} p<0.0014\right)$. Cumulatively, these results demonstrate that CNO administration decreases the excitability and activity of PFC PV cells expressing $h_{M 4 D G_{i}}$ both early during development and in adulthood.

\section{Developmental inhibition of PFC PV interneurons results in persistent alterations in behavior and prefrontal network functioning in adulthood.}

To test whether transiently inhibiting the activity of PFC PV cells during peripubertal and adolescent development results in persistent effects on behavior and prefrontal network function, we injected P10 PV-Cre pups with an AAV expressing hM4DG or mCherry and administered CNO between P14 and P50 (subsequently referred to as 'dev manipulation' or 'dev control', respectively; Figure 2A). We chose P14-50 because we wanted to start with a broad time window that includes maturation of both PFC PV intrinsic properties as well as the development and refinement of the quantity and strength of their synaptic connections (Miyamae et al., 2017, Yang et al., 2014, Le Magueresse and Monyer, 2013). We then tested the mice at least forty days following the last injection 
of $\mathrm{CNO}$ in an odor- and texture-based attentional set-shifting task (Figure 2B). In this task mice dig into two bowls filled with one of two different textures and scented with one of two different odors. Mice initially learn that odor but not texture predicts reward. During the extradimensional set-shift the mouse has to learn that the rule shifted from the odor dimension to the texture dimension. We and others have shown that the extradimensional shift is dependent on PFC PV interneurons (Canetta et al., 2016, Cho et al., 2015, Goodwill et al., 2018, Cho et al., 2020). We also implanted the mice with electrodes to record neural activity in the PFC during behavior to assess prefrontal network function.

We found that mice that experienced a transient manipulation of PFC PV interneuron activity during development were impaired in ED set-shifting as adults. Although these developmental manipulation mice performed the initial acquisition of the task comparably to their developmental control counterparts (Supplementary Figure 1A; $\mathrm{n}=8$ developmental control and 5 developmental manipulation mice, unpaired t-test, $\mathrm{p}=0.9822$ ), they took more trials to reach criterion during the ED set-shifting portion of the task (Figure $2 \mathrm{C} ; \mathrm{n}=8$ developmental control and 5 developmental manipulation mice, unpaired t-test, $\left.{ }^{* *} \mathrm{p}=0.0021\right)$. The increased number of errors in developmental manipulation animals was seen across both perseverative and random error types (Supplementary Figure 1B, $\mathrm{n}=6$ developmental control and 5 developmental manipulation mice, unpaired t-test, $\mathrm{p}=0.6042$; note: 2 developmental control mice did not make any errors). Mice did not differ in the time it took them to complete a trial, suggesting their impairment was not due to a decrease in motivation (Supplementary Figure 1C\&D; $n=8$ developmental control and 5 developmental manipulation mice, unpaired t-test, $p=0.5690$ for initial acquisition and $\mathrm{p}=0.9123$ for ED set-shifting).

Intriguingly we found that in developmental control animals, prefrontal power in the gamma frequency increased just prior to when the animals made their choice in the task (signified by digging in one of the pots), relative to gamma power recorded in the first 3 seconds of the task (here on referred to as 'baseline'; Figure 2D-E). We quantified gamma power $(40-90 \mathrm{~Hz})$ in the $3 \mathrm{~s}$ choice period just prior to the dig in the task and found that it was significantly elevated relative to baseline (Figure 2Eii; baseline $n=79$ trials from 8 dev control animals, task $n=88$ trials from $8 \mathrm{dev}$ control animals, mixed effects linear regression, choice versus baseline $\left.{ }^{* \star * *} p<0.0001\right)$. Importantly, there was no significant relationship between 40-90 Hz gamma power and running speed in our animals (Supplementary Figure 2A; $n=8$ dev control mice, one-way ANOVA with Geisser-Greenhouse correction, no effect of velocity $F(2.246,15.72)=2.066, p=0.1562)$, ensuring this task-induced increase in gamma power was not simply the result of altered velocity in the context of the choice period. Intriguingly, this increase in taskinduced gamma was seen only in trials in which the animal proceeded to make the correct choice and not the incorrect choice (Figure 2F\&Fi, baseline $n=79$ trials from $8 \mathrm{dev}$ control animals, correct=70 trials from $8 \mathrm{dev}$ control animals, incorrect=18 trials from $6 \mathrm{dev}$ control animals, mixed effects linear regression, correct versus baseline ${ }^{* * *} p<0.0001$, incorrect versus baseline $p=0.3473$; note 2 dev control mice did not have any incorrect trials). 
This difference in task-evoked gamma power prior to making a correct versus incorrect choice was not only seen at the whole group level. A machine learning algorithm trained solely on gamma power in this three second window prior to making a choice in a subset of 5 correct and 5 incorrect trials was capable of predicting the outcome of remaining trials on a trial by trial basis with an accuracy significantly greater than chance (Figure $2 F i i, n=55$ accurately predicted outcomes of 78 tested trials, binomial test ${ }^{* * *} p=0.0002$ ). It was not possible to predict trial outcome using other frequency ranges including 4-12 Hz and 90-120 Hz (Supplementary Figure 2B, $n=40$ accurately predicted outcomes of 78 tested trials, binomial test $p=0.4550$ for $4-12 \mathrm{~Hz}$ and $n=22$ accurately predicted outcomes of 78 tested trials, binomial test $p=0.9999$ for $90-120 \mathrm{~Hz}$ ).

This task-induced increase in gamma in correct, but not incorrect, trials was also present in animals that experienced a developmental manipulation of PFC PV interneurons (Figure 2G\&Gi, baseline $n=69$ trials from 4 dev manipulation animals, correct $n=46$ trials from 4 dev manipulation animals, incorrect $n=28$ trials from 4 dev manipulation animals, mixed effects linear regression, correct versus baseline ${ }^{* * *} p<0.001$, incorrect versus baseline $\mathrm{p}=0.0947$; note $1 \mathrm{dev}$ manipulation animal which was used for behavior did not produce usable data for physiology). However, the magnitude of the increase in correct trials relative to baseline was significantly smaller in developmental manipulation relative to control animals (Figure 2Gi, mixed effects linear regression, $\left.{ }^{* * * *} \mathrm{p}<0.0001\right)$. Moreover, in developmental manipulation animals, gamma power was not able to predict trial outcome with an accuracy significantly greater than chance $(n=27$ accurately predicted outcomes of 64 tested trials, binomial test $\mathrm{p}=0.9157)$.

\section{Adult inhibition of PFC PV interneurons does not lead to persistent alterations in behavior and prefrontal} network functioning in adulthood.

To determine whether P14-50 is a sensitive time window, we also assessed if there are persistent effects of transiently inhibiting PFC PV interneurons in adulthood by administering CNO to hM4DG $\mathrm{h}_{\mathrm{i}}$ and mCherryexpressing mice for a comparable length of time (P94-P130) in mature mice (referred to as 'adult manipulation' and 'adult control', respectively; Figure 3A). Neither ED set-shifting behavior nor prefrontal network function was significantly altered by manipulation of PFC PV interneuron activity during a window of comparable length in adulthood. Adult manipulation and control mice took a similar number of trials to reach criteria in both the initial acquisition (Supplemental Figure $3 \mathrm{~A}, \mathrm{n}=5$ adult control animals, 5 adult manipulation animals, unpaired t-test $p=0.6716$ ) and ED set-shifting portion of the task (Figure 3C; $n=5$ adult control animals, 5 adult manipulation animals, unpaired t-test $\mathrm{p}=0.3576$ ). There were no differences in the distribution of error types (perseverative or random, Supplemental Figure 3B; $n=5$ adult control animals, 5 adult manipulation animals, unpaired t-test $\mathrm{p}=0.2179$ ) and the latency to complete IA (Supplemental Figure $3 C ; n=5$ adult control animals, 5 adult manipulation animals, unpaired t-test $\mathrm{p}=0.688$ ) and ED set-shifting (Supplemental Figure 3D $\mathrm{n}=5$ adult control animals, 5 adult manipulation animals, unpaired t-test $p=0.2887$ ) was equivalent between the groups. We replicated the negative findings in the ED set-shifting behavior in a second cohort of mice where the virus was injected in adulthood, to assure the lack of behavioral effect following adult manipulation was not due to 
alterations in the levels of viral expression given the longer time elapsed between when the virus was injected and the CNO administered in adult manipulation versus developmental manipulation animals (Supplementary Figure 3E-G; $n=12$ adult control animals, $n=11$ adult manipulation animals, unpaired t-test, $p=0.1932$ for IA, $\mathrm{p}=0.8293$ for ED set-shift). In both adult control and manipulation mice, gamma power was induced in correct trials, but not incorrect trials, relative to baseline (Figure 3D \& Di; baseline $n=51$ trials from 5 adult control mice, correct=40 trials from 5 adult control mice, incorrect=12 trials from 5 adult control mice, mixed effects linear regression, correct versus baseline ${ }^{* * * *} p<0.0001$, incorrect versus baseline $p=0.3611$; Figure $3 E \& E i$; baseline $\mathrm{n}=44$ trials from 4 adult manipulation mice, correct=34 trials from 4 adult manipulation mice, incorrect=10 trials from 4 adult manipulation mice, mixed effects linear regression, correct versus baseline ${ }^{* * *} p<0.0001$, incorrect versus baseline $p=0.0604$ ). Moreover, the magnitude of the increase in correct trials relative to baseline was equivalent between adult control and manipulation mice (Figure $3 \mathrm{Ei}$, mixed effects linear regression, $p=0.481$ ) and the magnitude of the increase in correct trials relative to baseline was significantly greater in adult manipulation that developmental manipulation mice (mixed effects linear regression, $p=0.0128$ ). In both adult control and manipulation groups, gamma power was sufficient to predict trial outcome on a trial by trial basis with an accuracy significantly greater than chance (Figure 3Eii, n=28 accurately predicted outcomes of 42 tested trials for adult control, binomial test ${ }^{*} \mathrm{p}=0.0218$, and $n=24$ accurately predicted outcomes of 34 tested trials for adult manipulation, binomial test ${ }^{* *} \mathrm{p}=0.0122$ ). Cumulatively, these results demonstrate that peripuberty and adolescence is a sensitive period in which manipulations to the activity level of PFC PV interneurons lead to persistent alterations in ED set-shifting behavior and prefrontal induced gamma activity.

\section{Developmental inhibition of PFC PV interneurons results in persistent reductions in their functional inhibition of glutamatergic pyramidal cells in adulthood.}

Both ED attentional set-shifting and task-induced gamma activity depend on PV interneuron function. To determine whether developmental inhibition of PV interneurons impairs their functional integration into the mPFC network we utilized slice physiology. To this end, we used mice that genetically expressed Cre-recombinase, Cre-dependent channelrhodopsin 2 (ChR2) and Cre-dependent hM4DGi in PV interneurons. PV/ChR2/hM4DG mice were administered either CNO ('dev manipulation') or Saline as a control ('dev control') from P14-P50 (Figure 4A). Forty days following the end of the developmental manipulation (P90), mice were used for slice electrophysiology. Whole cell patch clamp recordings were made from pyramidal cells in layer II/III of PFC. The strength of GABAergic transmission from PV cells onto pyramidal cells was measured by evoking neurotransmitter release from PFC PV cells by delivering a $5 \mathrm{~ms}$ pulse of $473 \mathrm{~nm}$ blue light via the $40 x$ objective and recording the amplitude of the resulting light-evoked inhibitory post-synaptic currents (Le-IPSCs). Le-IPSC amplitudes were significantly reduced in developmental manipulation mice (Figure 4D, $n=11$ developmental control cells and 17 developmental manipulation cells, unpaired t-test, $\left.{ }^{*} p=0.032\right)$. We then determined whether this decrease in PV-interneuron mediated inhibition is reflected in the general degree of inhibition measured in pyramidal cells. To this end we measured the frequency and amplitude of spontaneous inhibitory post-synaptic currents (sIPSCs) that are derived from all inhibitory interneurons. sIPSC frequency was also significantly 
reduced in developmental manipulation mice relative to developmental control mice (Figure 4F, $n=12$ developmental control cells and 17 developmental manipulation cells, unpaired t-test, ${ }^{*} \mathrm{p}=0.0165$ ). In contrast, sIPSC amplitude was unchanged (Figure 4G, $p=0.8885$ ). Together, these results indicate that functional inhibition from PFC PV interneurons onto pyramidal cells is compromised following the developmental manipulation of PFC PV interneuron activity. The change in frequency, but not amplitude, further suggests a decrease in the quantity or functionality of presynaptic inputs.

\section{Developmental inhibition of PFC PV interneurons results in persistent reductions in the expression of molecular markers}

Decreased PFC PV presynaptic input onto pyramidal cells might result from multiple factors including 1) a loss of PV interneurons, 2) a loss of PV synaptic connections or 3) a physiological change in PV interneurons due to altered expression of PV (found in cell soma) or the GABAergic enzyme GAD65 (found in synaptic terminals). We conducted histological studies to investigate these three possibilities in developmental manipulation and control mice as adults (Supplementary Figure 4A). To address the first possibility, the number of PV containing cells was stereologically assessed in tissue sections containing the PFC. The number of PV-expressing cells per $\mathrm{mm}^{2}$ was not different between developmentally manipulated and control mice (Supplementary Figure 4B; $\mathrm{n}=7 \mathrm{dev}$ control and $9 \mathrm{dev}$ manipulation mice, un-paired t-test, $\mathrm{p}=0.4681$ ). To address the second possibility, we stained tissue for GAD65, which marks presynaptic GABAergic terminals, and assessed the number of GAD65 puncta in close proximity to pyramidal cell soma outlined using the cytoskeletal marker, MAP2. We found no differences in the size (Supplementary Figure 4C; $n=7$ dev control and 10 dev manipulation mice, unpaired t-test, 0.1333), number (Supplementary Figure 4D; $n=7 \mathrm{dev}$ control and $10 \mathrm{dev}$ manipulation mice, unpaired t-test, $\mathrm{p}=0.7074$ ) or intensity (Supplementary Figure 4E; $\mathrm{n}=7$ dev control and 10 dev manipulation mice, un-paired t-test, $\mathrm{p}=0.936$ ) of the perisomatic GAD65-expressing puncta. To address the third possibility, we stained tissue for PV, in combination with mCherry, to label virus-infected cells. We then quantified the intensity of PV staining in PV cells from developmental manipulation or control mice. We found that while the overall mean of PV staining was unchanged between these groups (Supplementary Figure 4F; $p=0.6844$ ), there was a trend towards a change in the overall distribution of the staining intensities, with an over-representation of lightlystained cells in the developmental manipulation group (Supplementary Figure 4G; Kolmogorov-Smirinov test, $\mathrm{p}=0.0627$ ). This is of interest as lightly stained PV neurons have recently been associated with reduced PV plasticity (Mukherjee et al., 2019).

\section{Enhancing PFC PV interneuron activity in adulthood with a SSFO can rescue behavioral deficits following developmental inhibition of PFC PV interneurons.}

Due to the importance of PFC PV interneurons for ED attentional set-shifting, we investigated whether we could rescue the deficits in ED set-shifting by acutely and selectively enhancing PFC PV interneuron excitability in adult mice, despite the fact that their functional connectivity is reduced after the developmental manipulation. Rather than stimulating neurons with ChR2, we decided to increase PV interneuron excitability using a stabilized 
step-function opsin (SSFO). This should enhance responsiveness of PV neurons to incoming endogenous activity without imposing an artificial stimulation pattern. P10 PV-Cre mice were stereotaxically injected in the PFC with either AAV5-hSyn-DIO-hM4DGi-mCherry or AAV5-hSyn-DIO-mCherry, in combination with AAV5EF1a-DIO-hChR2(C128S/D156A)-EYFP. CNO was administered between P14 and P50 for both developmental manipulation and developmental control mice. Seventy days following the end of each manipulation (P120) mice were evaluated in an attentional set-shifting task. Half the animals had the SSFO activated by administration of $473 \mathrm{~nm}$ light via bilaterally implanted optic fibers during ED set-shifting on testing day 1 . Ten days following the first evaluation of ED set-shift, testing was repeated with the SSFO being activated in the other half of the animals on testing day 2 (Figure 5A). Activation of PFC PV interneurons during ED set-shifting using a SSFO rescued behavioral deficits in mice that experienced a manipulation of PFC PV interneuron activity during development (Figure 5B-D). Application of light to acutely activate PFC PV cells led to a significant reduction in trials to criterion only in developmental manipulation, but not developmental control, mice (Figure 5D; $n=6$ developmental manipulation mice, 5 developmental control mice, 2-way rmANOVA, fixed effect of light $F(1,9)=10.55, p=0.01$; fixed effect of treatment $F(1,15)=0.3719, p=0.5511$; light by treatment interaction $F(1,9)=12.08, p=0.0053$; Bonferroni post-hoc developmental manipulation light $O N \vee$ OFF $\left.{ }^{* *} p=0.0009\right)$. These results show that artificially enhancing PFC PV interneuron activity is sufficient to rescue ED set-shifting behavior in developmental manipulation mice with compromised PFC PV interneuron function.

\section{Discussion}

\section{Identification of a postnatal sensitive period for PFC PV interneuron development and behavior}

Seminal studies in the visual system first identified sensitive periods in which changes in excitatory drive result in activity-dependent remodeling of thalamocortical inputs that become hard-wired, leading to long-lasting effects on visual functioning (Hensch, 2005, Antonini and Stryker, 1993, Hubel and Wiesel, 1970). Similar activitydependent remodeling has been shown for the refinement of inhibitory connections made by PV interneurons within the visual cortex during developmental sensitive periods (Baho and Di Cristo, 2012, Chattopadhyaya et al., 2007, Fu et al., 2012, Wu et al., 2012). A major result of our study is that activity-dependent sensitive periods also exist for prefrontal circuitry. Specifically, we found that transient inhibition of PFC PV interneurons has longlasting effects on their functional connectivity, prefrontal network function and behavior. The window we have initially identified for these effects spans peripuberty into adolescence (P14-P50). During this time, multiple aspects of PFC PV maturation typically occur including physiological alterations in their intrinsic membrane properties and firing properties, strengthening and refinement of their synaptic contacts within prefrontal circuitry, and increases in expression of activity-dependent proteins like PV itself (Caballero et al., 2020, Goodwill et al., 2018, Miyamae et al., 2017, Yang et al., 2014, Bitzenhofer et al., 2020). The dynamic nature of changes occurring to PFC PV cells in this time is consistent with the dynamic developmental windows that have been shown to be sensitive windows for other brain systems. 
At a behavioral level, we found that transiently decreasing the activity of PFC PV interneurons during this postnatal window resulted in persistent impairments in ED attentional set-shifting in adulthood, while manipulation of PFC PV interneuron activity for a comparable length of time in adulthood did not result in similar persistent behavioral effects. This is consistent with prior work demonstrating that ED set-shifting acutely depends on intact functioning of PFC PV interneurons in adult mice (Canetta et al., 2016, Cho et al., 2015, Goodwill et al., 2018, Cho et al., 2020). In related findings, Mukherjee et al. found that increasing activity of PFC PV interneurons during a late adolescent window (P60-P70) led to persistent rescue of set-shifting behavior in a genetic developmental risk factor mouse model (Mukherjee et al., 2019). However, that study left unclear if this late adolescent plasticity would be seen in typically developing animals in addition to genetically compromised ones. If manipulation of PV activity during both our peripubertal and early adolescent window and their late adolescent window was capable of eliciting long-lasting changes in set-shifting behavior, it would be important to understand if the neurobiological changes underlying the behavioral alterations produced by the manipulations during the different windows are comparable or disparate. Manipulations of PV activity in either window are associated with changes in expression of PV itself. However, Mukherjee et al. found that this decrease was associated with a decrease in excitatory inputs to PV interneurons, whereas we have found evidence that this phenotype is associated with a decrease in the strength of inhibition provided by these cells to neighboring pyramidal cells. Therefore, it is intriguing to speculate that while the function of PV cells may be compromised following manipulation in either window, the specific synaptic processes that are disrupted may differ.

\section{Identification of a task-induced gamma signal predictive of ED set-shifting performance}

How prefrontal PV interneurons influence prefrontal network function to enable ED set-shifting behavior remains an open question. We found that in control mice, the power of gamma frequency activity in the PFC increases while animals are preparing to make a choice in an ED set-shifting task, relative to a baseline familiar environment. Importantly, this increase is only seen in trials where they go on to make a correct, rather than an incorrect, choice. Prefrontal gamma frequency power is believed to reflect organization of the local pyramidal cell activity by PFC PV interneurons. Therefore, the observed task-induced increase in prefrontal gamma power suggests that PFC PV interneurons are normally engaged while the animal is preparing to make its choice in the ED set-shifting task. Interestingly, recent work from Cho et al. using in vivo calcium imaging to observe bulk changes in PFC PV interneuron activity during a comparable ED set-shifting task, suggests that PFC PV interneurons are also engaged in incorrect trials, once the animals realize they have made an incorrect choice (Cho et al., 2020). We did not observe significantly greater mean gamma frequency power after making an incorrect, rather than a correct, choice. However, we did observe a trend for the peak gamma frequency power to increase in incorrect trials after making a choice, relative to the peak in the period prior to the choice, while the opposite was true in correct trials. It may be that PFC PV interneurons transiently increase in activity following performance of an incorrect, but not correct, choice and that this transitory peak is better captured with cell-type 
selective fiber photometry, while gamma oscillations reflect changes in both PV interneurons as well as other elements of the local prefrontal network. Together, our results and those of Cho et al. provide several ways in which PFC PV interneurons may be engaged and required for different aspects of set-shifting behavior.

Gamma power was larger before correct choices in all control and experimental cohorts. However, the magnitude of this increase was significantly attenuated in developmental manipulation animals relative to developmental control animals. Practically, while gamma power could be used to predict trial outcome on a trial by trial basis with an accuracy significantly greater than chance in our developmental control, adult control and adult manipulation animals, this was not possible in our developmental manipulation mice. Cumulatively, our results suggest that under normal circumstances, PFC PV interneurons contribute to ED set-shifting behavior by organizing local pyramidal neuron activity in the gamma frequency as the animals prepare to make their choice in the task. This organizational capability may allow the flexible grouping/regrouping of neuronal ensembles representing different aspects of the task and stimulus environment, perhaps forming the neural basis for the changing stimulus-outcome associations necessary to guide proper task performance. Moreover, when the activity of these cells is manipulated during a sensitive window in postnatal development, it results in an impairment in the ability to engage the necessary gamma-frequency activity in the context of choice-related behavior and a subsequent reduction in ED set-shifting task performance. Future studies should use optogenetics to investigate the acute dependence of this prefrontal gamma signal on different cell populations within, as well as inputs to, the prefrontal cortex.

\section{Postnatal changes in PFC PV activity may alter their functional integration into cortical circuitry}

We also found that developmental suppression of PFC PV interneuron activity resulted in persistent reductions in the strength of the functional connections made onto local pyramidal cells. This finding is consistent with recent work showing that knockdown of prefrontal PV beginning in early adolescence persistently impaired the cells' inhibitory output in young adulthood (Caballero et al., 2020). The loss of inhibitory output following our developmental manipulation was not due to a loss of PV-expressing cells as stereological counts of PFC PV interneurons were unchanged in developmentally manipulated mice. Similarly, an analysis of GAD65-expressing perisomatic puncta indicates that the number of inhibitory synapses onto pyramidal cells is unchanged. Instead, our histological results identified a strong trend towards an overall decrease in the somatic expression of PV itself. This is interesting as PV is a calcium buffer whose expression both influences, and responds to, changes in PV cell activity. Decreased PV expression impairs calcium buffering, altering their release probability and impeding the ability of these cells to sustain firing at their characteristically high frequencies (Caillard et al., 2000). Moreover, normal increases in PV expression across development correspond with increases in the power and peak frequency of gamma oscillations (Bitzenhofer et al., 2020). Additionally, a shift towards lower PV intensity has been associated with reduced plasticity in those cells (Mukherjee et al., 2019). Finally, a reduction in PV 
expression has been seen in both animal models of genetic and environmental risk factors relevant to psychiatric disorders such as schizophrenia, as well as in post mortem observations from patients with schizophrenia (Hashimoto et al., 2003, Behrens et al., 2007, Belforte et al., 2010). Our results support the hypothesis that early changes in PV expression have long-lasting consequences for prefrontal network function.

Our findings comport with prior work in visual cortex demonstrating that alterations in the activity of PV interneurons during a developmental sensitive period can impact their integration into cortical circuitry (Chattopadhyaya et al., 2007, Fu et al., 2012, Baho and Di Cristo, 2012, Wu et al., 2012). However, in those studies, alterations in PV activity were primarily found to affect the stability of perisomatic inhibitory synapses made by those cells. In contrast, we find no evidence that our manipulation of PFC PV interneuron activity influences the number or size of perisomatic inhibitory synaptic contacts. These differences may be explained by our different methodologies for manipulating cell activity, the use of in vivo versus in vitro experiments, the sensitivity of the histological assays used to quantify synaptic contacts or differences between the visual and prefrontal cortex. Despite these potential differences in mechanism, both our work in prefrontal cortex, and prior work in visual cortex, indicates that alterations in activity levels of PV cells during discrete developmental sensitive periods, can lead to long-term consequences for the connectivity that these cells make within cortical circuitry. Given that prefrontal network activity is hypothesized to regulate the developmental refinement of prefrontal circuitry and function more broadly, in future studies it will be important to investigate how our manipulation of PFC PV activity influences the maturation of excitatory cortical connectivity (Chini and HanganuOpatz, 2020, Le Magueresse and Monyer, 2013).

\section{Activation of PFC PV interneurons can rescue ED set-shifting deficits in developmentally compromised mice}

Even though we found that mice that experienced a developmental manipulation of PFC PV cell activity show persistently impaired ED set-shifting behavior as well as impaired task-induced gamma oscillations, acutely enhancing PFC PV interneuron function in adulthood via use of a stabilized step-function-opsin was capable of rescuing behavior in these mice. This finding highlights the sufficiency of correcting the impairments in inhibition provided by PFC PV cells in developmental manipulation mice for rescuing behavior. Given that our developmental manipulation of PFC PV activity could potentially have resulted in changes in other cell populations in different brain regions due to downstream effects following chronic manipulation of these cells' activity, these results underscore the centrality of defects in PFC PV cells for the cognitive sequelae resulting from this developmental manipulation. Moreover, they give hope that targeted interventions might be effective for rescuing behavioral alterations in developmentally compromised adults.

Our results are similar to those of Cho et al, who found that targeted bilateral gamma-frequency stimulation of PFC PV interneurons rescued ED set-shifting deficits in adult mice carrying a genetic risk factor for interneuron 
dysfunction (Cho et al., 2015). Cho et al found that this gamma frequency stimulation actually led to persistent behavioral effects that resulted in a sustained rescue of animal behavior for up to ten days. We saw a trend towards a similar persistent effect of our endogenous enhancement of PFC PV activity, whereby the developmentally manipulated mice that experienced optical stimulation performed better ten days later when retested without optical stimulation than developmentally manipulated mice tested without optical stimulation that had no prior history of earlier optical stimulation in the context of the task $(p=0.13)$. However, these persistent effects should be interpreted cautiously given the small sample size as well as other differences between the groups that might be accounting for their differences in ED set-shifting performance. Nevertheless, the possibility of persistent effects following our rescue manipulation should be investigated in future studies, given that it raises the tantalizing possibility of long-lasting behavioral rescue in cognitively impaired individuals.

\section{Relevance to understanding developmental origins of schizophrenia}

Alterations in PFC PV interneurons are a hallmark of schizophrenia, a disorder which is also characterized by alterations in cognitive function such as impairments in ED attentional set-shifting (Lewis, 2014, Brown and Tait, 2016). Although schizophrenia typically emerges in late adolescence or early adulthood, it has a suspected developmental etiology due to a plethora of genetic and early environmental risk factors as well as the presence of attenuated symptoms prior to diagnosis (Lewis and Levitt, 2002). However, timing and mechanisms underlying this developmental vulnerability to disease remain unknown.

Our results indicate that developing PFC PV interneurons are particularly susceptible to changes in their activity levels during a period encompassing peripuberty and adolescence. If a comparable vulnerability exists in humans than these results would suggest that any manipulation that alters PFC PV activity could potentially lead to long lasting disruptions in the function of these cells as well corresponding impairments in cognitive behaviors like attentional set-shifting. Both behavioral-level manipulations, such as social interactions (Bicks et al., 2020), as well as molecular alterations, such as redox dysregulation (Steullet et al., 2017, Sullivan and O'Donnell, 2012), can affect PV activity. Consequently, our results indicate that the timing of exposure to risk factors like social isolation and oxidative stress may be extremely important in determining their long-term effects on behavior. Conversely, these findings suggest that therapeutic interventions delivered within this peripubertal and adolescent window might be particularly important in preventing later behavioral dysfunction in people at high risk for developing disease. Finally, our rescue experiments using targeted activation of PFC PV interneurons give hope that mechanistically oriented interventions may be able to improve developmentally-induced behavioral dysfunction in adulthood.

\section{Acknowledgements}


The authors thank Julia Greenwald for her assistance with animal husbandry. This work was supported by the Brain and Behavior Research Foundation (grant number 26089) to SEC and (grant number 27384) to AZH and the National Institute of Mental Health (grant number K01MH107760) to SEC, (grant number F31 MH119691) to LJB, (grant number K08MH109735) to AZH and (grant numbers R21 MH121334 and MH117454) to CK. Some figures were created using BioRender.com.

\section{Author Contributions}

Conceptualization, SEC and CK; Methodology, SEC and CK; Formal Analysis, SEC, ESH, LJB, AZH and RTO; Investigation, SEC, ESH, ET and LJB; Resources, SEC, AZH and CK; Writing - Original Draft, SEC and CK; Writing - Review and Editing, SEC, ESH, ET, LJB, RTO, AZH and CK; Supervision, SEC, AZH and CK; Funding Acquisition, SEC, AZH and CK.

\section{Declaration of Interests}

The authors declare no competing interests. 


\section{Figure Legends}

Figure 1. A chemogenetic system to transiently inhibit PFC PV interneurons during development or adulthood. (A) Illustration of viral expression in PFC-PV cells. Left shows expression of mCherry (left), PV (middle) or an overlay (right). (B) Schematic illustrating minimal (dark pink) and maximal (light pink) spread of virus among animals included in analysis. (C) Timeline of experiments for validating the function of hM4DG . Mice were injected with virus at P10, and whole cell patch clamp recordings were made at P35 and P115 from cells expressing hM4DGi-mCherry or mCherry at baseline and in response to bath application of $10 \mu \mathrm{M}$ CNO. (D) Representative traces illustrating hyperpolarization of the resting membrane potential of hM4DGi-expressing, but not mCherry-expressing, cells following bath application of CNO. (E) Quantification of CNO-induced hyperpolarization. mCherry expressing cells at P35 and P115 were pooled because CNO did not show an effect at either age. CNO-induced a significant hyperpolarization in P35 and P115-expressing hM4DGi-expressing cells relative to $\mathrm{mCherry-expressing} \mathrm{cells.} \mathrm{Dots} \mathrm{indicate} \mathrm{individual} \mathrm{cell} \mathrm{responses} \mathrm{and} \mathrm{bars} \mathrm{indicate} \mathrm{mean} \pm$ SEM. (F) Bath application of CNO decreased firing frequency as a function of input current for P35 PFC PV cells expressing hM4DGi, but not mCherry. $\mathrm{N}=6$ cells per group. (G) Bath application of CNO also decreased firing frequency as a function of input current for P115 PFC PV cells expressing hM4DGi. $N=5$ cells per group. Dots and lines in $\mathrm{F}$ and $\mathrm{G}$ depict mean response. Scale bar is $250 \mu \mathrm{m} .{ }^{*} \mathrm{p}<0.05,{ }^{* *} \mathrm{p}<0.01$.

\section{Figure 2. Developmental inhibition of PFC PV interneurons results in persistent alterations in prefrontal} network functioning and behavior in adulthood (related to Supplementary Figures 1 and 2). (A) Experimental schematic. P10 PV-Cre mice were stereotaxically injected with either AAV5-hSyn-DIO-hM4DGimCherry or AAV5-hSyn-DIO-mCherry in the PFC. CNO was administered between P14 and P50 to produce dev control (mCherry CNO) and dev manipulation ( $\mathrm{hM} 4 \mathrm{DG}, \mathrm{CNO})$ mice. Forty days following the end of each manipulation, P90 mice were evaluated in an attentional set-shifting task while local field potentials (LFPs) were simultaneously recorded in the PFC using an implanted electrode. (B) Illustration of behavioral and in vivo electrophysiology experiment. Mice were unilaterally implanted with electrodes to record LFPs in the PFC in the context of an ED attentional set-shifting task. (C) Mice that experienced a developmental manipulation of PFC PV interneuron activity from P14-P50 showed persistent impairments in ED set-shifting behavior when tested after P90. Dev manipulation mice take significantly more trials to reach criterion than dev controls. Dots indicate individual animal responses and bars indicate mean \pm SEM. (D) Heat map depicting normalized power (in artificial units, A.U.) as a function of frequency in the first 3 seconds of the task (baseline) and in a ten second window centered around when the dev control animals make their choice (signified by the dig, dotted red line). Note that gamma frequency activity $(40-90 \mathrm{~Hz})$ increases just before the animal makes its choice. (E) Normalized power as a function of frequency just prior to when the animal makes its choice in the task ('choice', blue line) versus in the first 3 seconds in the task ('baseline', black line) in dev control animals. Red shaded area indicates gamma frequency range $(40-90 \mathrm{~Hz})$. Lines and shading indicate mean \pm SEM. (i) Mean gamma power $(40-90$ 
$\mathrm{Hz}$ ) for all individual choice (blue) and baseline (black) sampling sessions for all of the dev control animals (AH). (ii) Mean gamma power for all individual baseline and choice sampling sessions color coded by the animal they were measured in. Gamma power is significantly elevated in the choice period relative to baseline. (F) Normalized power as a function of frequency showing this increase in gamma power just prior to when the animal makes its choice in ED set-shifting is only seen in trials in which the animal goes on to make a correct choice (green) and not an incorrect choice (orange) relative to baseline (black). Red shading indicates 40-90 Hz gamma range. Lines and shading indicate mean \pm SEM. (i) Mean gamma power $(40-90 \mathrm{~Hz})$ for all individual baseline and correct and incorrect choice sampling sessions color coded by the animal they were measured in. Gamma power is significantly elevated just prior to correct, but not incorrect, choices relative to baseline. Mean gamma power $(40-90 \mathrm{~Hz})$ in developmental control animals can predict choice outcome on a trial by trial basis with an accuracy significantly greater than chance. Bar indicates accuracy of model. Dotted line indicates chance level (50\%). (G) Same results from mice that experienced a developmental manipulation of PFC PV interneurons. (i) Although these mice also showed a significant increase in gamma prior to when the animal went on to make a correct choice, but not an incorrect choice, relative to baseline, the magnitude of this increase was significantly smaller than that of developmental control mice. (ii) Mean gamma power (40-90 $\mathrm{Hz})$ in developmental manipulation animals cannot predict choice outcome on a trial by trial basis with an accuracy significantly greater than chance. Bar indicates accuracy of model. Dotted line indicates chance level (50\%). ${ }^{* *} \mathrm{p}<0.01 ;{ }^{* * *} \mathrm{p}<0.001 ;{ }^{* * *} \mathrm{p}<0.0001$.

Figure 3. Adult inhibition of PFC PV interneurons does not result in persistent alterations in prefrontal network functioning and behavior in adulthood (related to Supplementary Figure 3). (A) Experimental schematic. P10 PV-Cre mice were stereotaxically injected with either AAV5-hSyn-DIO-hM4DGi-mCherry or AAV5-hSyn-DIO-mCherry in the PFC. CNO was administered between P94 and P130 to produce adult control (mCherry $\mathrm{CNO}$ ) and adult manipulation ( $\mathrm{hM} 4 \mathrm{DG}, \mathrm{CNO}$ ) mice. Forty days following the end of each manipulation P170 mice were evaluated in an attentional set-shifting task while LFPs were simultaneously recorded in the PFC using an implanted electrode. (B) Illustration of behavioral and in vivo electrophysiology experiment. Mice were unilaterally implanted with electrodes to record LFPs in the PFC in the context of an attentional set-shifting task. (C) Transient silencing of PFC PV interneurons in adulthood does not lead to long-lasting effects on setshifting behavior. Trials to criteria during extra-dimensional set-shifting are equivalent for mCherry and hM4DG mice treated with CNO from P94-P130 and tested at P170 (adult control and manipulation mice, respectively). Dots indicate individual animal responses and bars indicate mean \pm SEM. (D) Normalized power (artificial units, A.U.) as a function of frequency just prior to when the adult control mice make their choice in ED set-shifting is only seen in trials in which they go on to make a correct choice (green) and not an incorrect choice (orange) relative to baseline (black). Red shading indicates $40-90 \mathrm{~Hz}$ gamma range. Lines and shading indicate mean \pm SEM. (i) Mean gamma power $(40-90 \mathrm{~Hz})$ for all individual baseline and correct and incorrect choice sampling sessions for adult control mice color coded by the animal they were measured in. Gamma power is significantly 
elevated just prior to correct, but not incorrect, choices relative to baseline. (E) Similar increases in task evoked gamma only prior to correct (green), but not incorrect (orange), choices relative to baseline (black) were seen for adult manipulation mice. Lines and shading indicate mean \pm SEM. (i) Mean gamma power for all individual baseline and correct and incorrect choice sampling sessions for adult manipulation mice color coded by the animal they were measured in. Gamma power is significantly elevated just prior to correct, but not incorrect, choices relative to baseline. The magnitude of the increase in task-evoked gamma prior to correct choices, relative to baseline, was equivalent between the adult manipulation and control groups. (ii) Mean gamma power (40-90 Hz) in adult control and manipulation animals can predict choice outcome on a trial by trial basis with an accuracy significantly greater than chance. Bars indicate accuracy of the models. Dotted line indicates chance level $(50 \%)$. ${ }^{*} p<0.05,{ }^{* *} p<0.01,{ }^{* * *} p<0.0001$.

Figure 4. Developmental inhibition of PFC PV interneurons results in persistent reductions in their functional inhibition of glutamatergic pyramidal cells in adulthood (related to Supplementary Figure 4). (A) Schematic illustrating the strategy used to express $\mathrm{hM}^{\mathrm{D}} \mathrm{DG}$, and $\mathrm{ChR2}$ in PFC PV interneurons in young mice. PV-Cre mice also expressing Cre-dependent ChR2 were crossed with Cre-dependent hM4DG; mice. $\mathrm{PV} / \mathrm{ChR2} / \mathrm{hM} 4 \mathrm{DG}$ i mice were administered either CNO or Saline as a control from P14-P50. Forty days following the end of the developmental manipulation (P90), mice were used for slice electrophysiology. (B) Schematic illustrating the slice electrophysiology experiment. Whole cell patch clamp recordings were made from pyramidal cells in layer II/III of PFC from dev control and manipulation mice carrying ChR2 in their PV cells. The strength of GABAergic transmission from PV cells onto pyramidal cells was measured by evoking neurotransmitter release from PFC PV cells by delivering a $5 \mathrm{~ms}$ pulse of $473 \mathrm{~nm}$ blue light via the $40 \mathrm{x}$ objective and recording the amplitude of the resulting light-evoked inhibitory post-synaptic currents (Le-IPSCs). Spontaneous inhibitory post-synaptic currents (sIPSCs) were also recorded. (C) Representative traces showing Le-IPSCs from cells recorded from developmental control and developmental manipulation mice. (D) Le-IPSC amplitudes were significantly reduced in developmental manipulated mice. Dots indicate individual cell responses and bars indicate mean \pm SEM. (E) Representative traces showing sIPSCs from cells recorded from developmental control and developmental manipulation mice. (F) sIPSC frequency is significantly reduced in developmental manipulation mice relative to developmental control mice. Dots indicate individual cell responses and bars indicate mean \pm SEM. (G) sIPSC amplitude is unchanged. Dots indicate individual cell responses and bars indicate mean \pm SEM. ${ }^{*} p<0.05$.

Figure 5. Enhancing PFC PV interneuron activity in adulthood with a SSFO can rescue behavioral deficits following developmental inhibition of PFC PV interneurons. (A) Experimental schematic. P10 PVCre mice were stereotaxically injected in the PFC with either AAV5-hSyn-DIO-hM4DGi-mCherry or AAV5-hSynDIO-mCherry in combination with AAV5-EF1a-DIO-hChR2(C128S/D156A)-EYFP (SSFO). CNO was 
administered between P14 and P50 for both dev manipulation and dev control mice. Adult dev manipulation and control mice were implanted with optical fibers bilaterally over the PFC and seventy days following the end of each manipulation (P120) mice were evaluated in an attentional set-shifting task with and without optical activation of their PFC PV interneurons. (B) Example histology. hM4DGi-mCherry or mCherry (red) and SSFOEYFP (green) were expressed in PV cells in the PFC. Dotted lines denote bilateral placement of the fiberoptic implants. (C) Schematic illustrating the details of the cross-over experiment. Half the animals had the SSFO activated by administration of $473 \mathrm{~nm}$ light via bilaterally implanted optical fibers during ED set-shifting on testing day 1. 10 days following the first evaluation of ED set-shifting, testing was repeated with the SSFO being activated in the other half of the animals on testing day 2. (D) Following light activation of PFC PV cells, there was a significant reduction in the number of trials it took the dev manipulation animals to reach criterion in ED set-shifting but no change in dev controls. ${ }^{* *} \mathrm{p}<0.001$. Scale bar is $250 \mu \mathrm{m}$. 


\section{Experimental Procedures}

Animals All animal procedures were approved by Columbia University's Animal Care and Use Committee. C57/bl6 (Jackson Labs, Stock \#000664) mice were mated with Parvalbumin-Cre (PV-Cre, Jackson Stock \#008069) mice to produce heterozygous PV-Cre mice used for early viral injection experiments. For optogenetic slice electrophysiology experiments, mice heterozygous for PV-Cre and homozygous for Ai32 (Cre-dependent channelrhodopsin2 mice, Jackson Labs, Stock \#024109) were mated with RC::PDi homozygous mice (Credependent hM4DGi, gift of Susan Dymecki). Animals were fed ad libitum and reared under normal lighting conditions (12/12 light/dark cycle), unless otherwise noted. Adult (>P90) male and female mice were used for behavior, in vivo and in vitro electrophysiology and histology experiments (no effects of sex were seen).

Surgery Between postnatal day 9 and 12 (P9 to P12) young mice were anesthetized with a mixture of $40 \mathrm{mg} / \mathrm{kg}$ ketamine and $5 \mathrm{mg} / \mathrm{kg}$ xylazine and secured in a stereotax outfitted with a mouse pup adaptor using ear cuffs. Viruses, including AAV5-hSyn-DIO-hM4DG;-mCherry (\#44362, Addgene, 1.2x 10^13 titer), AAV5-hSyn-DIOmCherry (\#50459, Addgene, 4.8 x 10^12 GC/mL titer) and AAV5-Ef1a-DIO-hChR2(C128S/D156A)-EYFP (4.9x10^12 titer; UNC Vector Core, Chapel Hill, NC, USA), were injected bilaterally in the medial PFC (AP +0.92, $\mathrm{ML}+/-0.13$, DV -1.45 from skull at Bregma). $0.2 \mu \mathrm{l}$ of virus was injected at each site over the course of 2 minutes, followed by a 2-minute wait prior to withdrawing the injection pipette. A similar procedure was used to inject virus in adult mice. Animals were anesthetized with a mixture of $100 \mathrm{mg} / \mathrm{kg}$ of ketamine and $5 \mathrm{mg} / \mathrm{kg}$ of xylazine. Virus $(0.2 \mu \mathrm{l}$ per site followed by a 2-minute wait) was injected in the medial PFC with slightly modified coordinates (AP +1.8, ML +/- 0.35, DV -2.5 from skull at Bregma). Carprofen (1 mg/ml i.p., Zoetis, Parsippany, NJ, USA) was given for post-surgical analgesia. For implantation of the electrode bundle and/or optical stimulation fibers, adult animals were anesthetized with a mixture of $100 \mathrm{mg} / \mathrm{kg}$ of ketamine and $0.5 \mathrm{mg} / \mathrm{kg}$ of xylazine (i.p.), and administered $0.05 \mathrm{ml}$ of $1 \mathrm{mg} / \mathrm{ml}$ of dexamethasone (subcutaneously; Henry Schein, Melville, NY, USA) prior to surgery, to reduce brain swelling. Bupivacaine ( $5 \mathrm{mg} / \mathrm{ml}$; Hospira, Lake Forest, Illinois, USA) was also injected subcutaneously at the injection site as an additional analgesic. The electrode bundle composed of $76 \mu \mathrm{m}$ tungsten wire for the LFP and $13 \mu \mathrm{m}$ tungsten wire for the stereotrodes (California Fine Wire, Grover Beach, CA, USA) was implanted unilaterally in the left medial PFC (AP +1.8, ML +/- $0.35, D V-2.5$ from skull at Bregma). The electrode bundle connected to the microdrive (EIB-16 or EIB-32 narrow; Neuralynx, Bozeman, MT, USA) was fixed to a custom 3D-printed stage. Optic fibers were glued to the stage according to bilateral ML coordinates and the electrode bundle was glued to one of the fibers such that it extended $0.02 \mathrm{~mm}$ beyond the tip of the fiber.

Clozapine-n-oxide (CNO) was obtained from the $\mathrm{NIH}$ and stored at $-20{ }^{\circ} \mathrm{C}$. A $0.1 \mathrm{mg} / \mathrm{ml}$ working solution of CNO was prepared in $0.9 \%$ sterile saline at room temperature and administered at a concentration of $1 \mathrm{mg} / \mathrm{kg}$ (intraperitoneally, i.p.) twice daily from postnatal day 14 (P14) until P50 (developmental manipulation/control and 
developmental manipulation/control with SSFO) or P94 until P130 (adult manipulation/control). A new stock solution of CNO was prepared every two days and stored at room temperature, protected from light.

Attentional Set-Shifting Attentional set-shifting was performed as previously described (Canetta et al., 2016). Mice were food-deprived until they reached 80 to 85 percent of their baseline body weight. They received one day of habituation to the set-shifting enclosure (10 minutes foraging for pieces of honey-nut cheerios). That night they were habituated to the pots and the bedding media (corn cob and paper) used as part of the compound stimulus by placing the pots, filled with both types of bedding media as well as buried honey-nut cheerios, in their home cage. Mice then received training/shaping days, in which they were presented with two pots containing samples of either the unscented corn cob or paper bedding media used as part of the compound stimulus baited with a buried honey-nut Cheerio reward. Each day the animal received five trials, which continued until the animal found the buried Cheerio reward hidden in each pot. When the animal was consistently digging in each pot within 30 seconds to one minute following trial initiation, the next day they proceeded to behavioral testing. The first phase of testing was initial acquisition in which the mouse was presented with two pots containing a compound stimulus. The compound stimulus contained a combination of bedding media (corn cob or paper) with an odor (paprika or cinnamon) and only one dimension of the stimulus (odor, cinnamon) predicted the location of the buried Cheerio reward. For the first five trials of initial acquisition, the animals were allowed to dig in both pots to aid them in acquiring the rule, but the trials were scored as either correct or incorrect based on the first pot in which they chose to dig. Initial acquisition ended when the mice performed 8 out of 10 consecutive trials correctly. The total number of trials to reach this criteria, as well as the average latency per trial was analyzed. Once the mouse reached criteria in the initial acquisition portion of the task, it proceeded to the extra-dimensional set-shifting portion of the task. This phase of the task was identical to initial acquisition, except that the dimension of the stimulus that predicted the location of the food reward was changed (bedding media, paper). When the animal performed 8 of 10 consecutive trials correctly, testing was finished. For all trials, if the animal took longer than ten minutes to make a choice in a given trial, the trial was ended and recorded as an error. During ED set-shifting, errors resulting from the mouse making the choice that would have been rewarded in initial acquisition were called 'perseverative errors'. All other errors were called 'random errors'. For optogenetic stimulation experiments, set-shifting was performed twice. The first day it was performed as previously described. The second day (10 days after the first), paper bedding was the predictive stimulus in initial acquisition and in extra-dimensional set-shifting it switched to odor (paprika). The night prior to the second day of set-shifting, mice were re-introduced to pots containing unscented versions of both bedding medias baited with Cheerios in their home cage. No additional habituation or training was performed prior to the second setshifting test.

In vivo electrophysiology In vivo electrophysiology recordings were performed while the animals were performing the set-shifting task. Field potential signals from the medial PFC were referenced against a screw implanted in the anterior portion of the skull above the olfactory bulb. LFPs were amplified, bandpass filtered (1$1000 \mathrm{~Hz}$ ) and acquired at $2000 \mathrm{~Hz}$ with Lynx 8 programmable amplifiers on a personal computer running Cheetah 
data acquisition software (Neuralynx). The animal's position was obtained by overhead video tracking $(30 \mathrm{~Hz})$ of a LED affixed to the head stage. TTLs were manually inserted to record the timing of relevant events (e.g. trial start, dig, trial end).

Neuralynx files containing LFP data were imported into Matlab with Neuralynx MATLAB import/export package v 4.10. LFP samples were notch filtered using the MATLAB Chronux package to remove 60 cycle noise (http://chronux.org/; rmlinesmovingwinc.m). Mechanical artifacts were eliminated by removing samples whose voltage were more than 3 standard deviations from the entire signal mean. The cleaned signal was then rootmean-squared. Heat maps of normalized power (in artificial units, A.U.) as a function of frequency and time relative to the dig were constructed using the wavelet transformation package in Matlab (https://www.mathworks.com/help/wavelet/ref/cwt.html). Normalized power as a function of frequency was plotted by averaging the data from these plots in the relevant time windows (e.g. 3 seconds before dig for the choice period and the first 3 seconds of the trial for baseline; any trials less than $6 \mathrm{~s}$ long had their baseline excluded from the analysis). Mean gamma was calculated for the range of $40-90 \mathrm{~Hz}$. Electrode locations were confirmed to be within the medial PFC based on location of electrolytic lesions.

Optogenetic stimulation Optical stimulation was provided by a laser emitting blue light (473 $\mathrm{nm})$ at $4 \mathrm{~mW}$ connected via optical fibers $(200 \mu \mathrm{m}, 0.22 \mathrm{NA})$ to the light fibers $(200 \mu \mathrm{m}, 0.22 \mathrm{NA}$, average $80 \%$ transmittance) implanted in the animals' heads to activate the stabilized step-function-opsin (SSFO). Mice were randomized to receive light ON or OFF in a counterbalanced fashion on one of the two set-shifting test days. During testing, mice received a 5-second pulse of blue light when they were in the familiar environment just prior to beginning extra-dimensional set-shifting. Mice then received a 5-second pulse of blue light every thirty minutes to maintain the SSFO channels in an open state until testing was complete. All light pulses were administered in the familiar environment between trials.

Slice Electrophysiology Whole-cell current and voltage clamp recordings were performed in layer $2 / 3$ pyramidal cells and fast-spiking parvalbumin-expressing (PV) interneurons in the prelimbic region of the medial prefrontal cortex. Recordings were obtained with a Multiclamp 700B amplifier (Molecular Devices) and digitized using a Digidata 1440A acquisition system (Molecular Devices) with Clampex 10 (Molecular Devices) and analyzed with pClamp 10 (Molecular Devices). Following decapitation, $300 \mu \mathrm{M}$ slices containing the PFC were incubated in artificial cerebral spinal fluid (ACSF) containing (in mM) $126 \mathrm{NaCl}, 2.5 \mathrm{KCl}, 2.0 \mathrm{MgCl}, 1.25$ $\mathrm{NaH} 2 \mathrm{PO} 4,2.0 \mathrm{CaCl}$, 26.2 $\mathrm{NaHCO} 3$ and 10.0 D-Glucose, bubbled with oxygen, at $32^{\circ} \mathrm{C}$ for 30 minutes before being returned to room temperature for at least 30 minutes prior to use. During recording, slices were perfused in ACSF (with drugs added as detailed below) at a rate of $5 \mathrm{~mL} /$ minute. Electrodes were pulled from $1.5 \mathrm{~mm}$ borosilicate-glass pipettes on a P-97 puller (Sutter Instruments). Electrode resistance was typically 3-5 M $\Omega$ when filled with internal solution consisting of (in mM): $130 \mathrm{~K}$-Gluconate, $5 \mathrm{NaCl}, 10 \mathrm{HEPES}, 0.5 \mathrm{EGTA}, 2 \mathrm{Mg}-\mathrm{ATP}$, and $0.3 \mathrm{Na}-\mathrm{GTP}$ (pH 7.3, $280 \mathrm{mOsm})$. 
Effects of CNO on intrinsic and active membrane properties. hM4DGi-mCherry or mCherry-infected PV cells in the medial PFC were identified by their fluorescence at 40x magnification under infrared and diffusion interference contrast microscopy using an inverted Olympus BX51W1 microscope coupled to a Hamamatsu C8484 camera. Intrinsic and active membrane properties (resting membrane potential, input-output firing frequency curve) were recorded in current clamp using the K-Gluconate intracellular solution detailed above before and after $10 \mu \mathrm{M}$ CNO was bath applied to the slice.

Le-IPSC and SIPSC Recordings. Pyramidal cells were visually identified based on their shape and prominent apical dendrite at 40x magnification under infrared and diffusion interference contrast microscopy using an inverted Olympus BX51W1 microscope coupled to a Hamamatsu C8484 camera. Light-evoked postsynaptic inhibitory currents (Le-IPSCs) and spontaneous inhibitory postsynaptic currents (sIPSCs) were recorded in voltage clamp at a holding potential of $-70 \mathrm{mV}$ using a high-chloride intracellular solution containing (in $\mathrm{mM}$ ): 140 $\mathrm{CsCl}, 4 \mathrm{NaCl}, 1 \mathrm{MgCl}_{2}$, 10 HEPES, 0.05 EGTA, 2 ATP Mg${ }^{2+}$, and $0.4 \mathrm{GTP} \mathrm{Mg}^{2+}$ (pH 7.3, $\left.280 \mathrm{mOsm}\right) .20 \mu \mathrm{M} 6-$ cyano-7-nitroquinoxaline-2,3-dione disodium salt (CNQX, Tocris Bioscience, Briston, UK) and $50 \mu \mathrm{M}$ D-(-)-2amino-5-phosphonopentanoic acid (AP5, Tocris Bioscience) were added to the bath to block glutamatergic currents. The cells were placed in the center of the field of view, held at $-70 \mathrm{mV}$ in voltage clamp and the current response evoked by a 5 -ms pulse of blue light $(473 \mathrm{~nm})$ applied by a light emitting diode (LED, Cool LED, Andover, UK) was recorded. The intensity of the LED was set at $1 \%$ of maximum intensity. The current trace was filtered with an eight-pole low-pass Bessel filter and the difference between the baseline and the maximum light-evoked current response was recorded. sIPSCs were assessed from 60 seconds of the current recording at a holding potential of $-70 \mathrm{mV}$ filtered with an eight-pole low-pass Bessel filter and detected using MiniAnalysis (Synaptosoft, Fort Lee, NJ, USA). All event data was averaged by cell.

Histology Adult mice were deeply anesthetized with $100 \mathrm{mg} / \mathrm{kg}$ ketamine and $5 \mathrm{mg} / \mathrm{kg}$ xylazine (i.p.) and perfused with phosphate-buffered saline followed by $4 \%$ paraformaldehyde in phosphate-buffered saline. Brains were dissected out and post-fixed in 4\% paraformaldehyde overnight before being transferred to $1 \%$ PBS for long-term storage. Brains were sectioned serially at $50 \mu \mathrm{m}$ on a vibratome (Leica, Buffalo Grove, IL, USA). The following primary antibodies were used: Parvalbumin (PV; Sigma, Saint Louis, MO, USA, P3088, 1:2000), glutamate decarboxylase 65 (GAD65; Millipore, Billerica, MA, USA, MAB351, 1:1000), microtubule-associated protein (MAP2; Abcam, ab5392, 1:5000), mCherry (rabbit-anti-dsRed; Takara Bio, Mountainview, CA, USA; 632496, 1:500) or green fluorescent protein (GFP; Abcam, Cambridge, UK, ab13970, 1:1000). Primary antibody incubation was 48 hours at $4^{\circ} \mathrm{C}$. Alexa Fluor-conjugated secondary antibodies (Invitrogen, 1:1000) were used for secondary detection. Stereology was used to assess PV cell number in the prelimbic region of adult developmental manipulation and control offspring using Stereolnvestigator software (MBF Biosciences, Williston, VT, USA) on a Zeiss epifluorescence microscope (Carl Zeiss Microscopy, LLC, White Plains, NY, USA). GAD65 perisomatic puncta as well as levels of PV present in hM4DGi-mCherry and mCherry-expressing cells in the prelimbic region of the PFC were estimated from analysis of 10x images acquired on a Leica confocal microscope (Leica Microsystems, Buffalo Grove, IL, USA) of sections stained for either PV and mCherry or 
GAD65 and MAP2, using Image $\mathrm{J}$ software (NIH). During image acquisition and quantification, the investigator was blind to the treatment.

Analysis Statistical analysis and graph preparation of all data except the in vivo electrophysiology was done with Prism 8 software (Graphpad Software, San Diego, CA). In vivo electrophysiology analysis was done with custom scripts in MATLAB (Mathworks, Natick, MA) or R (https://www.r-project.org/) and graph preparation of this data was done in MATLAB or Statview (SAS Institute, Cary, NC, USA). To analyze differences in gamma power across animals, trials, and groups, we fit linear mixed models with gamma power as outcome. The random effect was animal, and fixed effects were trial (baseline, correct, or incorrect) and group (dev control, dev manipulation, adult control, or adult manipulation). To analyze the relationship between velocity and gamma power, velocity and mean gamma were evaluated in $50 \mathrm{~ms}$ bins during the entirety of the task. The mean gamma power within velocity bins of $0-7,7-15,15-21$ and $21-30 \mathrm{~cm} / \mathrm{s}$ was calculated for each animal and compared with a one-way rmANOVA. For predictor analysis, the fitclinear command from the MATLAB machine learning toolbox was used. The mean gamma power for each trial was used as a predictor and choice for each trial (correct vs incorrect) was transformed into binary vectors. To avoid over representation of correct trials, equal numbers of either correct or incorrect choice trials were randomly selected to train the model (10 total, 5 correct and 5 incorrect). This model was then tested on the remaining trials and the probability of achieving the observed number of successes given a theoretical distribution based on $50 \%$ accuracy was determined with a binomial test. 


\section{Supplemental Figure Legends}

\section{Supplementary Figure 1 (related to Figure 2). Additional behavioral analysis relevant to the PFC PV} developmental manipulation. (A) Trials to reach criterion in the initial acquisition phase of the set-shifting task is not affected by developmental manipulation of PFC PV interneurons. (B) There is no difference in the percentage of perseverative errors made by developmental manipulation mice. Developmental manipulation and control animals have a comparable mean latency to make a choice in both the initial acquisition (C) as well as extra-dimensional phase (D) of the set-shifting task. In all graphs, dots show individual animal values and bars show the mean \pm SEM.

\section{Supplemental Figure 2 (related to Figure 2). Additional electrophysiological analysis relevant to PFC PV} developmental manipulation. (A) Mean gamma power $(40-90 \mathrm{~Hz}$, analyzed for developmental control animals) does not significantly vary as a function of running speed. Dots show mean values for each individual animal and bars show the mean \pm SEM. (B) 4-12 and 90-120 Hz mean power cannot predict trial outcome in developmental control animals with an accuracy significantly greater than chance. Bar indicates accuracy. Dotted line indicates chance level (50\%).

\section{Supplemental Figure 3 (related to Figure 3). Additional behavioral analysis relevant to PFC PV adult} manipulation. (A) Trials to reach criterion in the initial acquisition phase of the set-shifting task is not affected by adult manipulation of PFC PV interneurons. (B) There is no difference in the percentage of perseverative errors made by adult manipulation mice. Adult manipulation and control animals have a comparable mean latency to make a choice in both the initial acquisition (C) as well as extra-dimensional phase (D) of the setshifting task. (E) Schematic illustrating timeline for adult manipulation experiment where the virus is injected at $\mathrm{P} 80$ rather than $\mathrm{P} 10$. CNO was administered between P94 and P130 and behavior was assessed from P170 onwards. (F) Adult manipulation animals with virus injected at P80 rather than P10 still take a comparable number of trials to reach criterion in both the initial acquisition $(\mathbf{F})$ as well as extra-dimensional phase $(\mathbf{G})$ of the setshifting task as adult controls. In all graphs, dots show individual animal values and bars show the mean \pm SEM.

\section{Supplementary Figure 4. Developmental inhibition of PFC PV interneurons does not alter PV cell and} synapse number but trends towards decreasing somatic PV levels. (A) Experimental schematic. P10 PVCre mice were stereotaxically injected with either AAV5-hSyn-DIO-hM4DG;-mCherry or AAV5-hSyn-DIOmCherry in the PFC. CNO was administered between P14 and P50 for the developmental manipulation and developmental control mice. At least forty days following the end of each manipulation, mice were processed for histology. (B) Stereological counts of PV-expressing cells in the PFC of developmental control and developmental manipulation mice. The number of PV-expressing cells did not differ between these two groups. (C) The size, (D) number and (E) intensity of GAD65-expressing perisomatic puncta did not differ between developmental control and developmental manipulation mice. (F) The overall mean intensity of PV in virusinfected cells did not differ between developmental control and developmental manipulation mice. (G) There was 
bioRxiv preprint doi: https://doi.org/10.1101/2021.03.04 433943; this version posted March 4, 2021. The copyright holder for this preprint (which was not certified by peer review) is the author/funder, who has granted bioRxiv a license to display the preprint in perpetuity. It is made available under aCC-BY-NC-ND 4.0 International license.

a trend towards a difference in the distribution of the intensity of PV expression in virus-infected cells in developmental control and developmental manipulation mice. In graphs B-E, dots show individual animal values and bars show the mean \pm SEM. In graph $\mathrm{F}$, dots show individual cell values and bar shows the mean \pm SEM. 


\section{References}

ANTONINI, A. \& STRYKER, M. P. 1993. Rapid remodeling of axonal arbors in the visual cortex. Science, 260, 1819-21.

BAHO, E. \& DI CRISTO, G. 2012. Neural activity and neurotransmission regulate the maturation of the innervation field of cortical GABAergic interneurons in an age-dependent manner. $J$ Neurosci, 32, 911 8.

BEHRENS, M. M., ALI, S. S., DAO, D. N., LUCERO, J., SHEKHTMAN, G., QUICK, K. L. \& DUGAN, L. L. 2007. Ketamine-induced loss of phenotype of fast-spiking interneurons is mediated by NADPH-oxidase. Science, 318, 1645-7.

BELFORTE, J. E., ZSIROS, V., SKLAR, E. R., JIANG, Z., YU, G., LI, Y., QUINLAN, E. M. \& NAKAZAWA, K. 2010. Postnatal NMDA receptor ablation in corticolimbic interneurons confers schizophrenia-like phenotypes. Nat Neurosci, 13, 76-83.

BICKS, L. K., YAMAMURO, K., FLANIGAN, M. E., KIM, J. M., KATO, D., LUCAS, E. K., KOIKE, H., PENG, M. S., BRADY, D. M., CHANDRASEKARAN, S., NORMAN, K. J., SMITH, M. R., CLEM, R. L., RUSSO, S. J., AKBARIAN, S. \& MORISHITA, H. 2020. Prefrontal parvalbumin interneurons require juvenile social experience to establish adult social behavior. Nature Communications, 11.

BIRRELL, J. M. \& BROWN, V. J. 2000. Medial frontal cortex mediates perceptual attentional set shifting in the rat. Journal of Neuroscience, 20, 4320-4324.

BISSONETTE, G. B., MARTINS, G. J., FRANZ, T. M., HARPER, E. S., SCHOENBAUM, G. \& POWELL, E. M. 2008. Double dissociation of the effects of medial and orbital prefrontal cortical lesions on attentional and affective shifts in mice. $J$ Neurosci, 28, 11124-30.

BITZENHOFER, S. H., POPPLAU, J. A. \& HANGANU-OPATZ, I. 2020. Gamma activity accelerates during prefrontal development. Elife, 9.

BOSCHIN, E. A., MARS, R. B. \& BUCKLEY, M. J. 2017. Transcranial magnetic stimulation to dorsolateral prefrontal cortex affects conflict-induced behavioural adaptation in a Wisconsin Card Sorting Test analogue. Neuropsychologia, 94, 36-43.

BROWN, V. J. \& TAIT, D. S. 2016. Attentional Set-Shifting Across Species. Curr Top Behav Neurosci, 28, 36395.

CABALLERO, A., FLORES-BARRERA, E., THOMASES, D. R. \& TSENG, K. Y. 2020. Downregulation of parvalbumin expression in the prefrontal cortex during adolescence causes enduring prefrontal disinhibition in adulthood. Neuropsychopharmacology, 45, 1527-1535.

CAILLARD, O., MORENO, H., SCHWALLER, B., LLANO, I., CELIO, M. R. \& MARTY, A. 2000. Role of the calcium-binding protein parvalbumin in short-term synaptic plasticity. Proceedings of the National Academy of Sciences of the United States of America, 97, 13372-13377.

CANETTA, S., BOLKAN, S., PADILLA-COREANO, N., SONG, L. J., SAHN, R., HARRISON, N. L., GORDON, J. A., BROWN, A. \& KELLENDONK, C. 2016. Maternal immune activation leads to selective functional deficits in offspring parvalbumin interneurons. Mol Psychiatry, 21, 956-68.

CARDIN, J. A., CARLEN, M., MELETIS, K., KNOBLICH, U., ZHANG, F., DEISSEROTH, K., TSAI, L. H. \& MOORE, C. I. 2009. Driving fast-spiking cells induces gamma rhythm and controls sensory responses. Nature, 459, 663-U63.

CHATTOPADHYAYA, B., DI CRISTO, G., WU, C. Z., KNOTT, G., KUHLMAN, S., FU, Y., PALMITER, R. D. \& HUANG, Z. J. 2007. GAD67-mediated GABA synthesis and signaling regulate inhibitory synaptic innervation in the visual cortex. Neuron, 54, 889-903.

CHINI, M. \& HANGANU-OPATZ, I. L. 2020. Prefrontal Cortex Development in Health and Disease: Lessons from Rodents and Humans. Trends in Neurosciences.

CHO, K. K., HOCH, R., LEE, A. T., PATEL, T., RUBENSTEIN, J. L. \& SOHAL, V. S. 2015. Gamma rhythms link prefrontal interneuron dysfunction with cognitive inflexibility in Dlx5/6(+/-) mice. Neuron, 85, 133243.

CHO, K. K. A., DAVIDSON, T. J., BOUVIER, G., MARSHALL, J. D., SCHNITZER, M. J. \& SOHAL, V. S. 2020. Cross-hemispheric gamma synchrony between prefrontal parvalbumin interneurons supports behavioral adaptation during rule shift learning. Nat Neurosci. 
FU, Y., WU, X., LU, J. \& HUANG, Z. J. 2012. Presynaptic GABA(B) Receptor Regulates Activity-Dependent Maturation and Patterning of Inhibitory Synapses through Dynamic Allocation of Synaptic Vesicles. Front Cell Neurosci, 6, 57.

GLASCHER, J., ADOLPHS, R. \& TRANEL, D. 2019. Model-based lesion mapping of cognitive control using the Wisconsin Card Sorting Test. Nat Commun, 10, 20.

GOODWILL, H. L., MANZANO-NIEVES, G., LACHANCE, P., TERAMOTO, S., LIN, S., LOPEZ, C., STEVENSON, R. J., THEYEL, B. B., MOORE, C. I., CONNORS, B. W. \& BATH, K. G. 2018. Early Life Stress Drives Sex-Selective Impairment in Reversal Learning by Affecting Parvalbumin Interneurons in Orbitofrontal Cortex of Mice. Cell Rep, 25, 2299-2307 e4.

HASHIMOTO, T., VOLK, D. W., EGGAN, S. M., MIRNICS, K., PIERRI, J. N., SUN, Z., SAMPSON, A. R. \& LEWIS, D. A. 2003. Gene expression deficits in a subclass of GABA neurons in the prefrontal cortex of subjects with schizophrenia. J Neurosci, 23, 6315-26.

HENSCH, T. K. 2005. Critical period mechanisms in developing visual cortex. Current Topics in Developmental Biology, Vol 69, 69, 215-+.

HU, H., GAN, J. \& JONAS, P. 2014. Fast-spiking, parvalbumin(+) GABAergic interneurons: From cellular design to microcircuit function. Science, 345, 529-+.

HUBEL, D. H. \& WIESEL, T. N. 1970. Period of Susceptibility to Physiological Effects of Unilateral Eye Closure in Kittens. Journal of Physiology-London, 206, 419-\&.

LE MAGUERESSE, C. \& MONYER, H. 2013. GABAergic Interneurons Shape the Functional Maturation of the Cortex. Neuron, 77, 388-405.

LEWIS, D. A. 2014. Inhibitory neurons in human cortical circuits: substrate for cognitive dysfunction in schizophrenia. Current Opinion in Neurobiology, 26, 22-26.

LEWIS, D. A. \& LEVITT, P. 2002. Schizophrenia as a disorder of neurodevelopment. Annual Review of Neuroscience, 25, 409-432.

MARKRAM, H., TOLEDO-RODRIGUEZ, M., WANG, Y., GUPTA, A., SILBERBERG, G. \& WU, C. 2004. Interneurons of the neocortical inhibitory system. Nat Rev Neurosci, 5, 793-807.

MIYAMAE, T., CHEN, K., LEWIS, D. A. \& GONZALEZ-BURGOS, G. 2017. Distinct Physiological Maturation of Parvalbumin-Positive Neuron Subtypes in Mouse Prefrontal Cortex. J Neurosci, 37, 4883-4902.

MUKHERJEE, A., CARVALHO, F., ELIEZ, S. \& CARONI, P. 2019. Long-Lasting Rescue of Network and Cognitive Dysfunction in a Genetic Schizophrenia Model. Cell, 178, 1387-1402 e14.

ROTH, B. L. 2016. DREADDs for Neuroscientists. Neuron, 89, 683-94.

SMITH, E. E. \& JONIDES, J. 1999. Storage and executive processes in the frontal lobes. Science, 283, 165761.

SOHAL, V. S., ZHANG, F., YIZHAR, O. \& DEISSEROTH, K. 2009. Parvalbumin neurons and gamma rhythms enhance cortical circuit performance. Nature, 459, 698-702.

STEULLET, P., CABUNGCAL, H., COYLE, J., DIDRIKSEN, M., GILL, K., GRACE, A. A., HENSCH, T. K., LAMANTIA, S., LINDEMANN, L., MAYNARD, T. M., MEYER, U., MORISHITA, H., O'DONNELL, P., PUHL, M., CUENOD, M. \& DO, K. Q. 2017. Oxidative stress-driven parvalbumin interneuron impairment as a common mechanism in models of schizophrenia. Molecular Psychiatry, 22, 936-943.

SULLIVAN, E. M. \& O'DONNELL, P. 2012. Inhibitory Interneurons, Oxidative Stress, and Schizophrenia. Schizophrenia Bulletin, 38, 373-376.

UHLHAAS, P. J. \& SINGER, W. 2010. Abnormal neural oscillations and synchrony in schizophrenia. Nat Rev Neurosci, 11, 100-13.

WONDERS, C. P. \& ANDERSON, S. A. 2006. The origin and specification of cortical interneurons. Nat Rev Neurosci, 7, 687-96.

WU, X., FU, Y., KNOTT, G., LU, J., DI CRISTO, G. \& HUANG, Z. J. 2012. GABA signaling promotes synapse elimination and axon pruning in developing cortical inhibitory interneurons. J Neurosci, 32, 331-43.

YANG, J. M., ZHANG, J., YU, Y. Q., DUAN, S. \& LI, X. M. 2014. Postnatal development of 2 microcircuits involving fast-spiking interneurons in the mouse prefrontal cortex. Cereb Cortex, 24, 98-109.

ZHANG, L. I. \& POO, M. M. 2001. Electrical activity and development of neural circuits. Nat Neurosci, 4 Suppl, 1207-14. 
Figure 1.

A.
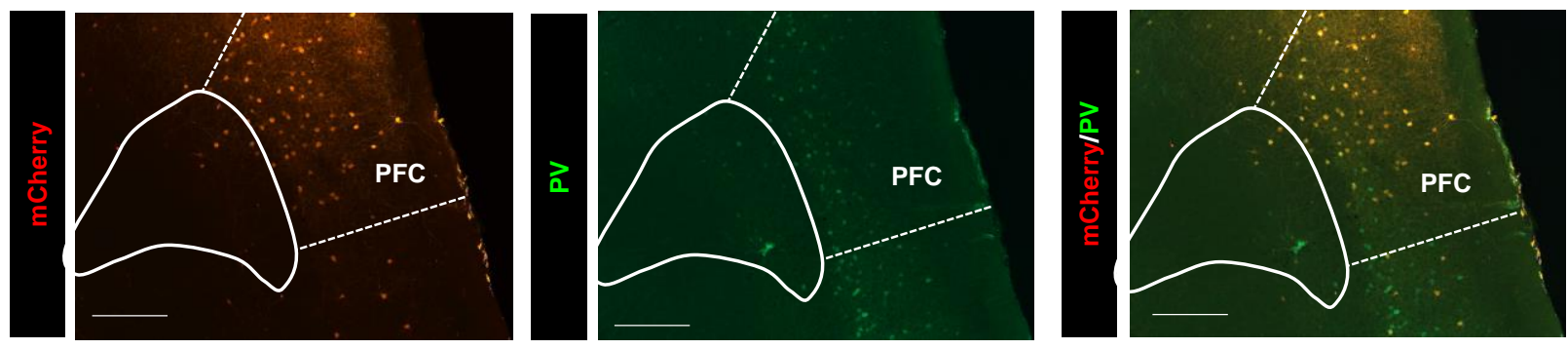

B.

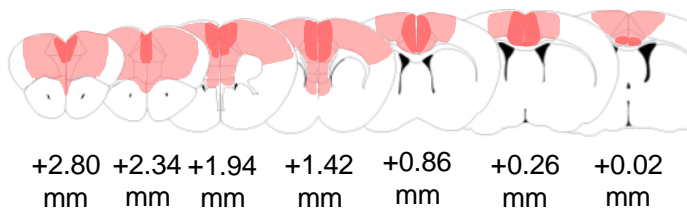

D.

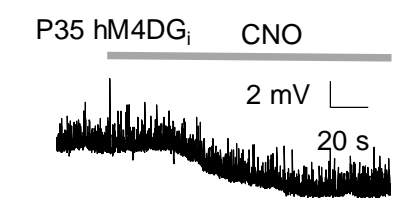

P115 hM4DG

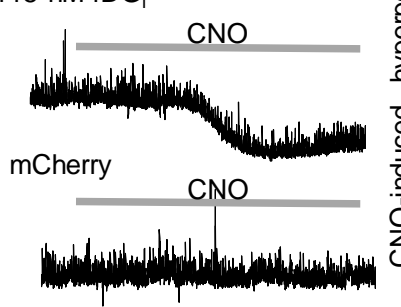

E.

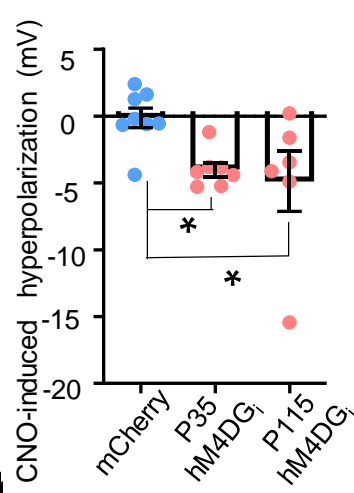

C.
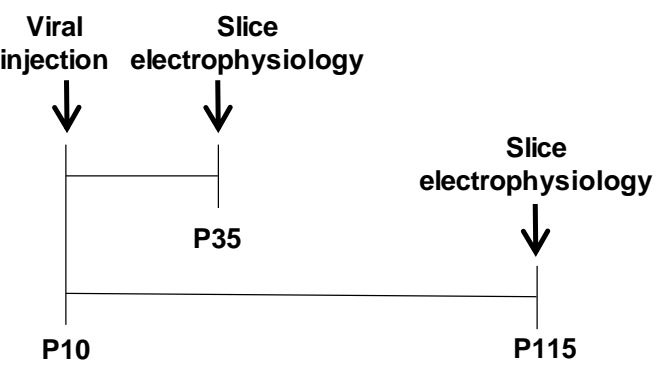

F.

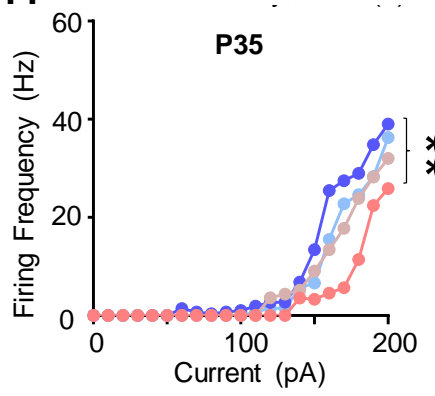

G.

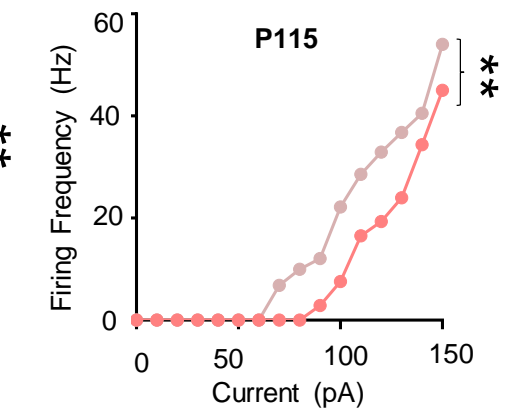

$\rightarrow \mathrm{hM} 4 \mathrm{DG} \mathrm{i}_{\mathrm{i}} \mathrm{ACSF} \rightarrow \mathrm{mCherry}$ ACSF

$\rightarrow \mathrm{hM} 4 \mathrm{DG} \mathrm{G}_{\mathrm{i}} \mathrm{CNO} \quad \rightarrow$ mCherry CNO 
Figure 2.

A.

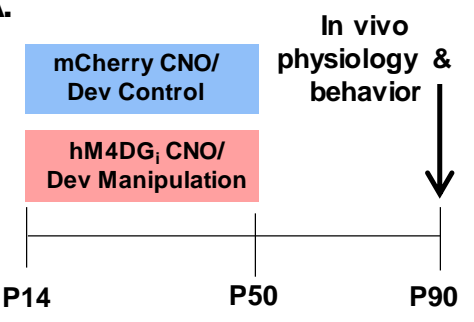

Attentional Set-Shifting

B. Initial Acquisition ED Set-Shift
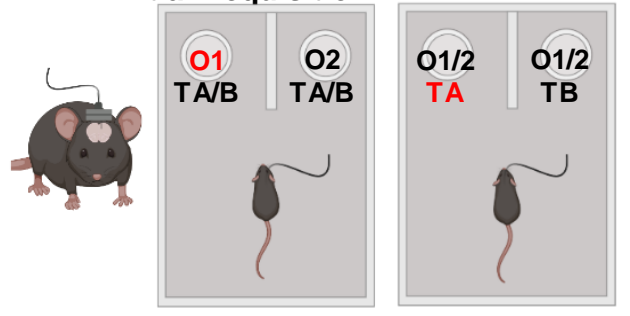

C. ED Set-Shift Dev

C. Dev Control

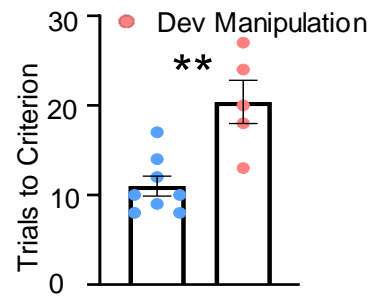

D.

E. 0.5

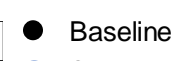

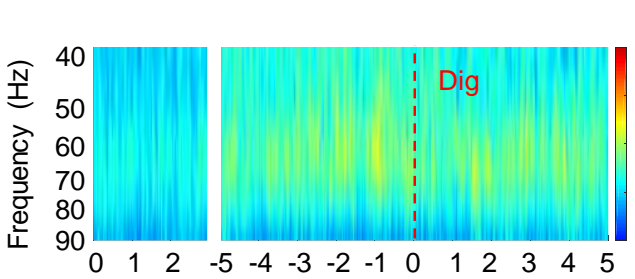
Baseline (s)

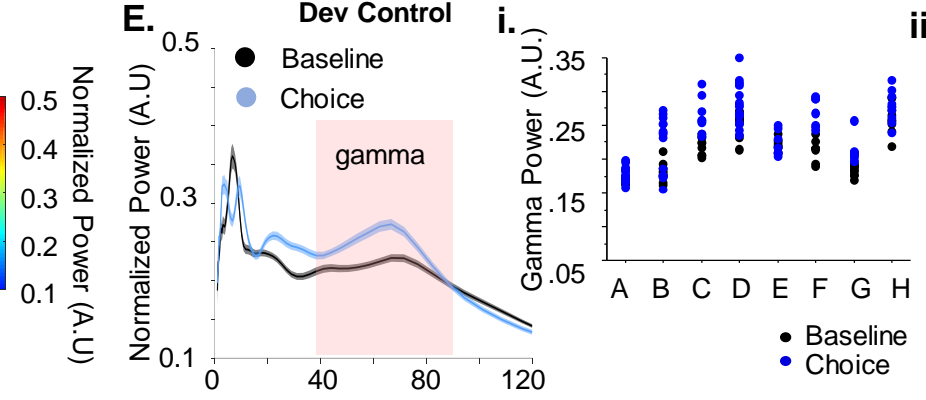

- Choice

ii.

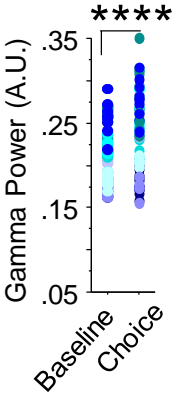

Frequency

i. $* * * *$ ii.

F.

$-35 \stackrel{* * * *}{1 i .}$
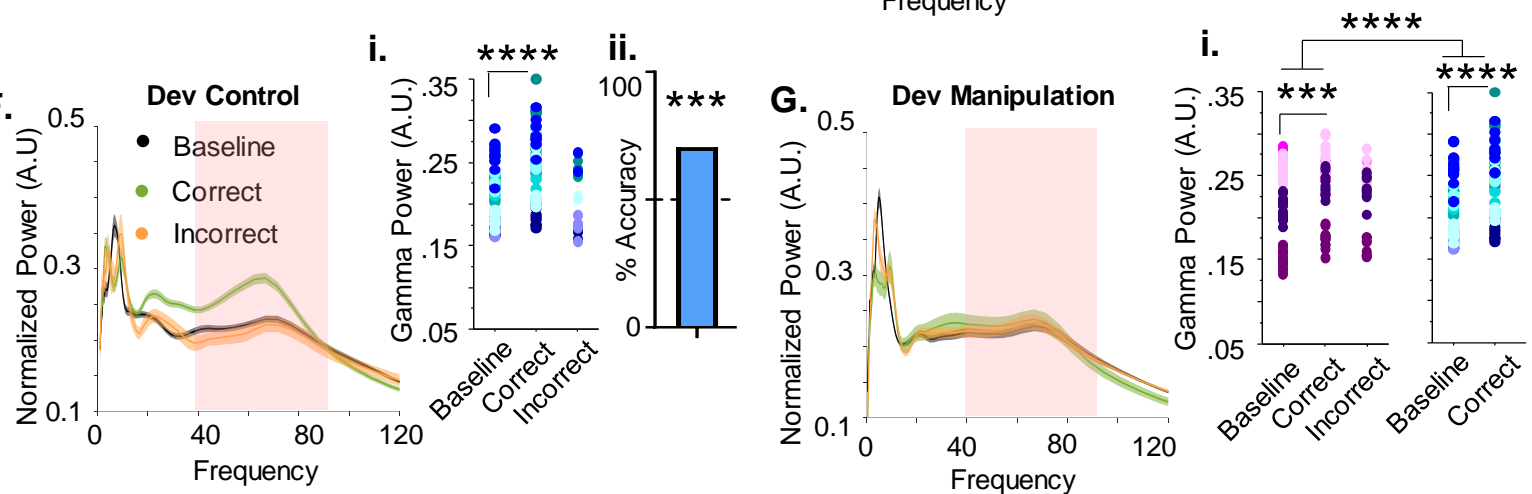

ii.

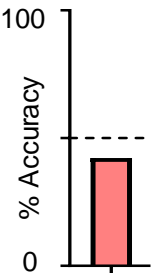


Figure 3.

A.

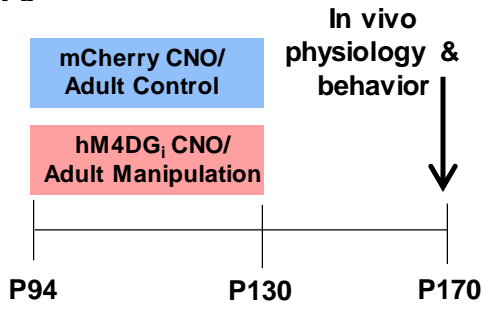

B.
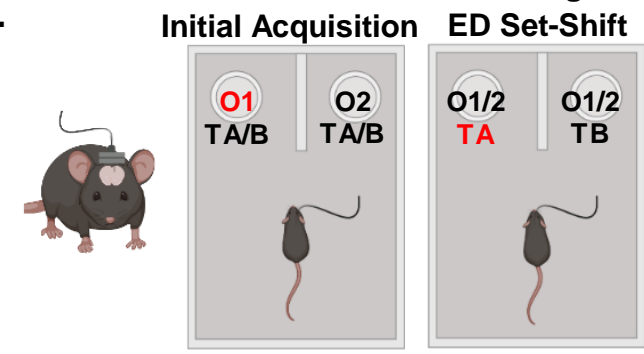

C. ED Set Shift Adult

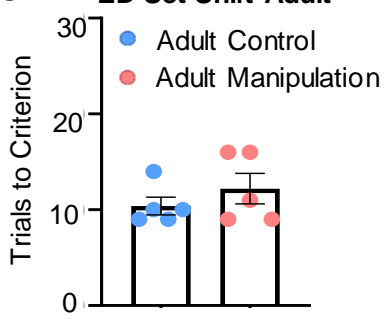

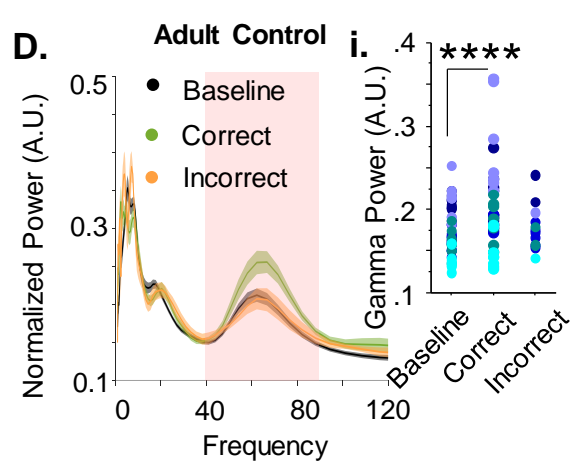

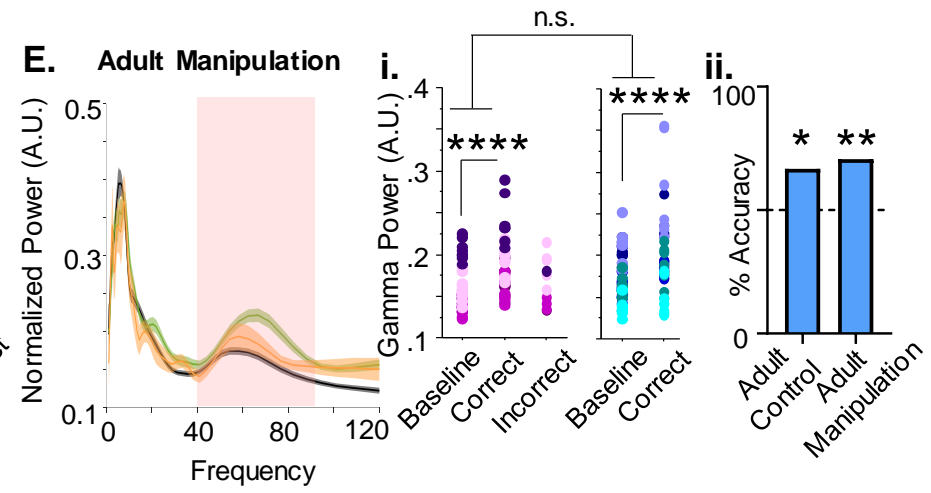


Figure 4.

A.

B. $\square \quad 1 \times 5 \mathrm{~ms}$ pulse

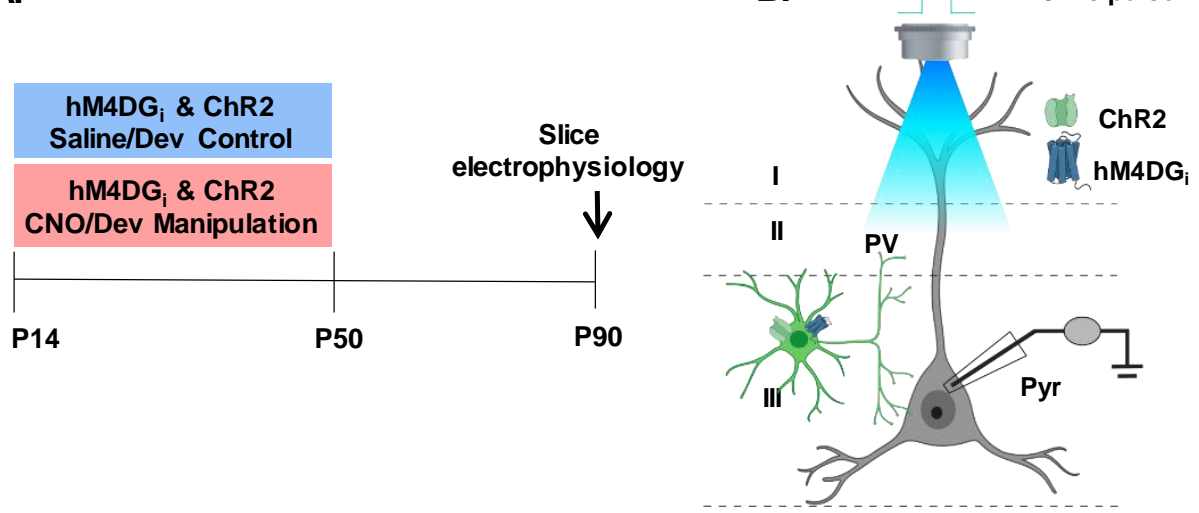

C. Dev Control Dev Manipulation

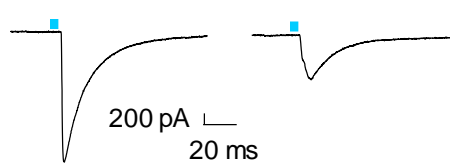

E.

Dev Control

Dev Manipulation
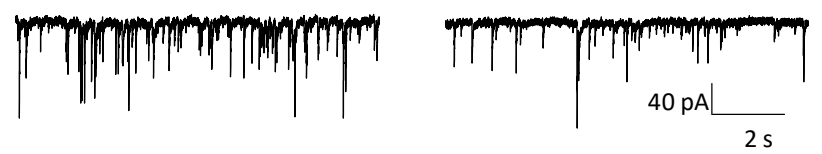

D.

F.

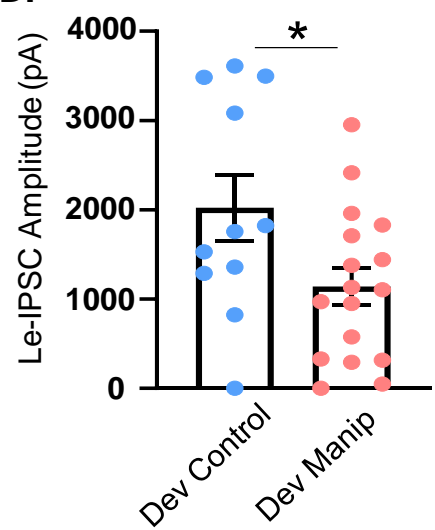

G.

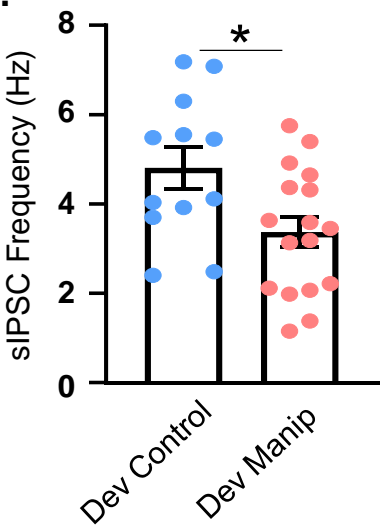

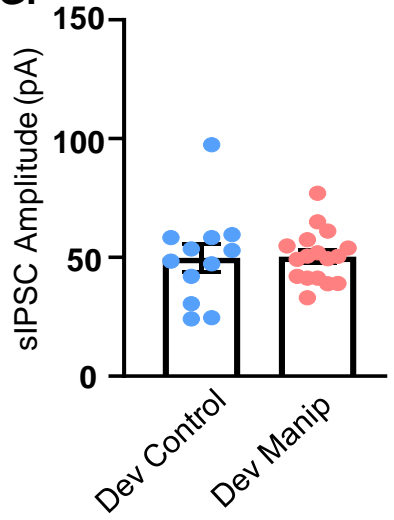


Figure 5.

A.
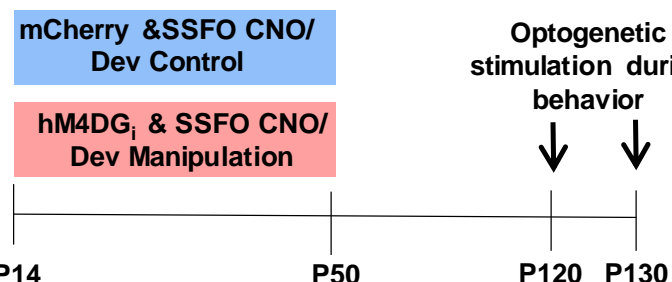

P14
Optogenetic stimulation during behavior

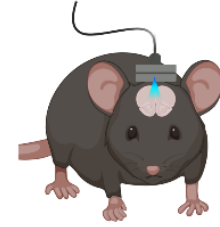

B.

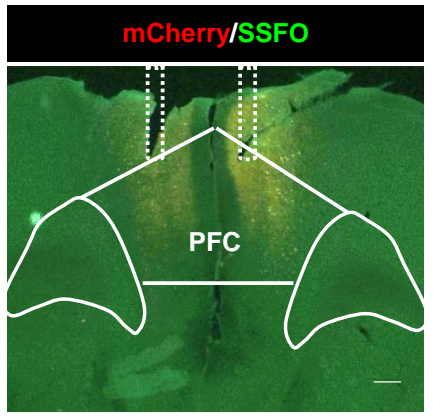

D.

ED Set-Shifting

P130/D2 Rescue: Half On/Half Off

5s pulses, $470 \mathrm{~nm}$ light

Initial Acquisition

0102

TA/B $30 \mathrm{~min}$

TA/B

P120/D1 Rescue: Half On/Half Off

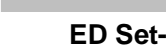
Sh"ting

\begin{tabular}{ll}
$01 / 2$ & $01 / 2$ \\
TB & TA \\
\hline
\end{tabular}

5s pulses, $470 \mathrm{~nm}$ light

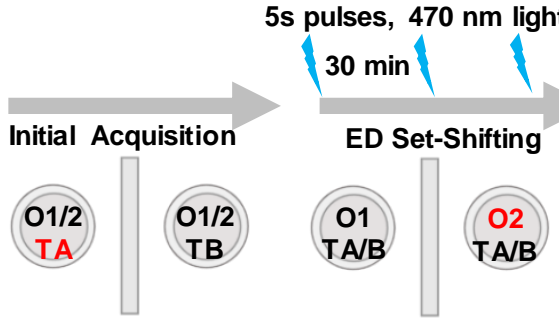

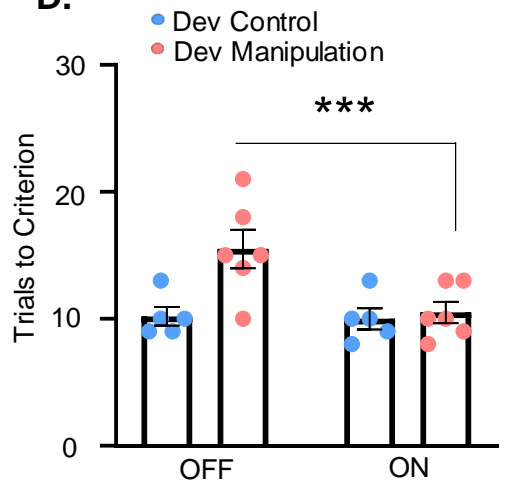

